\title{
INTERACTION OF MASSLESS FERMIONS WITH INSTANTONS ${ }^{\star}$
}

\author{
Choonkyu LEE \\ Department of Physics, The University of Michigan, Ann Arbor, Michigan 48109, USA \\ William A. BARDEEN \\ Theoretical Physics Department, Fermi National Accelerator Laboratory, Batavia, Illinois \\ 60510, USA
}

Received 7 February 1979

\begin{abstract}
We develop a systematic method of calculating the massless fermion propagation function and fermion-induced effective action in the background gauge field of an arbitrary number of widely separated instantons and anti-instantons. The results exhibit a strongly non-local nature of instanton interactions with massless fermions. Using these results, we also demonstrate that the ordinary perturbation theory vacuum is not allowed in QCD with massless fermions, since the cluster decomposition for certain correlation functions is violated due to widely separated instanton-anti-instanton bound states. The underlying mechanism is identical to that in the Schwinger model.
\end{abstract}

\section{Introduction and summary}

In QCD, it is well-known that presence of massless fermions completely suppresses the tunnelling phenomenon between topologically distinct winding number vacua unless fermion pairs are created [1-3]. In Euclidean space-time description, this becomes manifest by the presence of normalizable zero eigenmodes for the fermion field equation operator in a background gauge field of nonzero Pontryagin number [1,4]. Also, Callan, Dashen and Gross argued [5] that the exchange of massless fermions gives rise to a logarithmic attractive interaction between instantons and anti-instantons. These phenomena simply indicate that the complete treatment of instanton interactions in massless QCD should involve the simultaneous study of the gauge field action, the effective action due to vacuum polarization of fermions, and the propagation of various fermion operators. (This is precisely the procedure which has been taken in the two-dimensional QED - the Schwinger model [6].)

\footnotetext{
* Supported in part by the Department of Energy.
} 
As the first step of such a scheme, in this paper we develop a systematic method of calculating the massless fermion propagation function and the fermion-induced effective action in the given background gauge field of an arbitrary number of widely separated instantons and anti-instantons. We will work entirely in Euclidean space-time and assume the $\mathrm{SU}(2)$ gauge group with $N_{\mathrm{f}}$ fermion isodoublets $\left(N_{\mathrm{f}}\right.$ : number of flavours). Construction of the fermion propagation function becomes quite delicate when the zero eigenmodes originally associated with individual (anti-) instantons gain small "energy" and become nonnormalizable (i.e., plane waves asymptotically). It goes without saying that extreme care is necessary in dealing with interference between normalizable modes and the non-normalizable continuum. We resolve this difficulty by expressing the full fermion propagation function in such a background gauge field in terms of the fermion zero-modes and propagation functions (in the non-zero-mode subspace) in the individual (anti-) instanton potentials. The fermion-induced effective action can be obtained from the fermion propagation function by using the standard variational formula. The massless fermion propagation function and fermion-induced effective action exhibit a strongly non-local nature of instanton interactions with massless fermions. We also clarify the precise nature of the so-called zero-modc approximation $[2,13]$.

Using the propagation function and the fermion-induced effective action thus constructed, we analyze the cluster decomposition property in massless QCD [2]. We demonstrate that, in the ordinary perturbation theory vacuum, the cluster decomposition for the correlation functions involving operators of non-zero chirality is violated due to widely separated instanton-anti-instanton field configurations. The underlying mechanism is identical to that in the Schwinger model. Strong logarithmic attraction between an instanton and an anti-instanton, which is generated by vacuum polarization of massless fermions, is balanced by strong repulsion due to explicit fermion propagations. Analogous behavior in the Schwinger model has been noted by Nielsen and Schroer [7]. Here, we should mention that the cluster decomposition property in massless QCD has been considered in ref. [2], but without due considerations (at least in the paper) of the strongly non-local nature of instanton interactions with massless fermions. The breakdown of cluster decomposition is resolved by going to the so-called $|\theta\rangle$ vacua [2]. Implications of our results to a variety of instanton-related problems, for example the spontaneous breakdown of chiral $\mathrm{SU}(N)$ symmetry, are under investigation.

The plan of our paper is as follows. In sect. 2 we construct the fermion propagation function in the background field of an arbitrary number of widely separated instantons and anti-instantons in a systematic way. In sect. 3 , we evaluate the fermion-induced effective action in such a background gauge field. We discuss the cluster decomposition in massless QCD in sect. 4. In the appendix, we discuss some properties of the fermion zero modes and illustrate the absence of normalizable fermion zero modes in a simple instanton-anti-instanton system. 


\section{Fermion propagation function}

In this section, we develop a systematic method of calculating the fermion propagation function in the background gauge field

$$
\begin{aligned}
A_{\mu}^{a}(x) & =\sum_{i=1}^{n} A_{\mu i}^{a}\left(x, z_{i}\right) \\
& =\sum_{i=1}^{n_{+}} A_{\mu+}^{a}\left(x-z_{i}\right)+\sum_{i=n_{+}+1}^{n} A_{\mu-}^{a}\left(x-z_{i}\right),
\end{aligned}
$$

where $A_{\mu \pm}^{a}\left(x \quad z_{i}\right)(a=1,2,3)$ denotes a localized gauge field configuration with the center at $x=z_{i}$ and the Pontryagin number $w= \pm 1$, i.e., $A_{\mu+}^{a}$ for an instanton and $A_{\mu-}^{a}$ for an anti-instanton. (In this paper, we use a generic name, (anti-) instanton, to indicate any localized gauge field with $w=+1(-1)$, not just the classical (anti-) instanton solutions first found in ref. [8]. The most well-known examples of such non-trivial localized gauge fields with $w= \pm 1$ are the singular gauge expressions of the BPST solutions [8]

$$
\boldsymbol{A}_{\mu \pm}^{\mathrm{cl}}(x-z) \cdot \frac{1}{2} \boldsymbol{\tau}=\mathrm{e}^{-i \boldsymbol{\Omega} \cdot \tau / 2} \frac{1}{g} \frac{\eta_{\mu \nu a}^{(\mp)}(x-z)_{\nu} \tau_{a} \rho^{2}}{(x-z)^{2}\left[(x-z)^{2}+\rho^{2}\right]} \mathrm{e}^{i \boldsymbol{\Omega} \cdot \tau / 2},
$$

where the $\eta$-symbols are defined by [9] $(\mu, \nu=1,2,3,4$ and $k, l=1,2,3)$

$$
\eta_{\mu \nu a}^{( \pm)}=-\eta_{\nu \mu a}^{( \pm)}, \quad \eta_{k l a}^{( \pm)}=\epsilon_{k l a}, \quad \eta_{k 4 a}^{( \pm)}= \pm \delta_{k a} .
$$

We assume that instantons and anti-instantons in the field (1) are widely separated from one another. Assuming that fermion zero-mode wave functions and propagation functions (in the non-zero-mode subspace) in individual (anti-) instanton fields are known, we shall construct the full fermion propagation function in the background gauge potential (1) in terms of those functions. The difficulty of this construction lies in how to disentangle interactions between normalizable zero modes and non-normalizable continuum. In particular, great care should be taken when both instantons and anti-instantons are present, since certain linear combinations of fermion zero modes associated with individual instantons or anti-instantons join the continuum in a non-perturbative way (see the appendix).

Let us first summarize our knowledge about a single (anti-) instanton. 't Hooft noted [1] that, with the exact classical solution (2) as the gauge potential, there exists a normalizable solution to

$$
\gamma_{\mu} D_{\mu} \psi_{0}=\gamma_{\mu}\left(\partial_{\mu}-i g \boldsymbol{A}_{\mu} \cdot \frac{1}{2} \tau\right) \psi_{0}=0
$$

with $\gamma_{5} \psi_{0 \pm}=\mp \psi_{0 \pm}$. (We use the $\gamma$-matrix representation satisfying $\left\{\gamma_{\mu}, \gamma_{\nu}\right\}=-$ $2 \delta_{\mu \nu}, \gamma_{\mu}^{+}=-\gamma_{\mu}$ and $\gamma_{5}=\gamma_{1} \gamma_{2} \gamma_{3} \gamma_{4}$.) Explicitly [1],

$$
\psi_{0 \pm}(x-z)=\mathrm{e}^{-i \boldsymbol{\Omega} \cdot \tau / 2}\left(\frac{\sqrt{2} \rho}{\pi}\right) \frac{-i \tau_{\mu}^{( \pm)}(x-z)_{\mu}}{\left[(x-z)^{2}\right]^{1 / 2}\left[(x-z)^{2}+\rho^{2}\right]^{3 / 2}} \chi_{ \pm},
$$


with $\tau_{\mu}^{( \pm)}=(\tau, \pm i)$ and the constant vector $\chi_{ \pm}$specified by

$$
\chi_{ \pm}^{\dagger} \chi_{ \pm}=1, \quad \chi_{ \pm} \chi_{ \pm}^{\dagger}=\frac{1}{8}\left(1 \mp \gamma_{5}\right)-\frac{1}{16} i \tau_{a} \eta_{\mu \nu a}^{( \pm)} \gamma_{\mu} \gamma_{\nu} .
$$

According to a general theorem [4], if $N_{ \pm}$denotes a number of normalizable fermion zero modes with $\gamma_{5}^{\prime}- \pm 1$, then

$$
N_{+}-N_{-}=-w,
$$

for each fermion flavour. In this paper, we will further assume that $N_{+}=0$ for a single instanton and $N_{-}=0$ for a single anti-instanton. We also note that the zeromode wave functions (5) behave asymptotically

$$
\psi_{0 \pm}(x-z) \underset{|x-z|>\rho}{\longrightarrow} \mathrm{O}\left(\frac{1}{|x-z|^{3}}\right)
$$

Though the general proof is lacking, we believe that the asymptotic behavior (8) is quite generally true with any sufficiently localized gauge field of $w= \pm 1$. To support this assertion, more general field configurations other than the exact classical solution (2) and associated fermion zero modes are considered in the appendix. From now on, we will thus assume the following for a localized single (anti-) instanton described by $A_{\mu \pm}^{a}(x-z)$,

(i) $w= \pm 1$,

(ii) $A_{\mu \pm}^{a}(x-z) \underset{|x-z| \rightarrow \infty}{\longrightarrow} \mathrm{O}\left(1 /|x-z|^{3}\right)$ or vanishes faster,

(iii) $\gamma_{\mu} D_{\mu}$ possesses a single zero mode $\psi_{0 \pm}\left(x-z_{i}\right)$ satisfying

$$
\begin{aligned}
& \gamma_{5} \psi_{0 \pm}(x-z)=\mp \psi_{0 \pm}(x-z), \\
& \psi_{0 \pm}(x-z) \underset{|x-z| \rightarrow \infty}{\longrightarrow} \mathrm{O}\left(\frac{1}{|x-z|^{3}}\right) .
\end{aligned}
$$

The condition (ii) requires the gauge potential to be in the singular gauge.

Due to presence of a normalizable zero mode, the massless fermion propagation function in a single (anti-) instanton field cannot be defined. Thus, we may regulate the zero eigenvalue by introducing a small fermion mass $m$ and solve the Green function equation [10]

$$
\left(\gamma_{\mu} D_{\mu}+i m\right) S_{ \pm}=I
$$

where we employ the operator notations, $S(x, y)=\langle x|S| y\rangle, \delta^{4}(x-y)=\langle x|I| y\rangle$, etc. Rearranging the fermion propagation function, we may write [10]

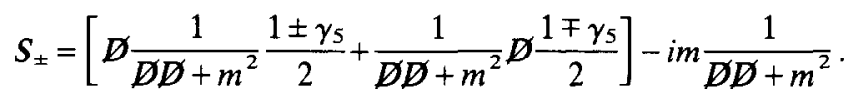

We now note that, due to projection operators $\frac{1}{2}\left(1 \pm \gamma_{5}\right)$, the terms inside the 
square bracket in eq. (11) have a well-defined zero-mass limit [9]

$$
S_{ \pm}^{\prime}=\not D \frac{1}{D D} \frac{1 \pm \gamma_{5}}{2}+\frac{1}{\not D D} \not D \frac{1 \mp \gamma_{5}}{2}
$$

On the other hand,

$$
-i m \frac{1}{\not D D+m^{2}} \underset{m \rightarrow 0}{\longrightarrow} \frac{\left|\psi_{0 \pm}\right\rangle\left\langle\psi_{0 \pm}\right|}{i m}+\mathrm{O}(m),
$$

where $\left|\psi_{0 \pm}\right\rangle$ denotes the fermion zero mode discussed earlier. Using the representation (12), it is a simple matter to show that [9]

$$
\begin{aligned}
& \gamma_{\mu} D_{\mu} S_{ \pm}^{\prime}=I-\left|\psi_{0 \pm}\right\rangle\left\langle\psi_{0 \pm}\right| \\
& {\left[S_{ \pm}^{\prime}\right]^{\dagger}=S_{ \pm}^{\prime}} \\
& \left\langle\psi_{0 \pm}\left|S_{ \pm}^{\prime}=S_{ \pm}^{\prime}\right| \psi_{0 \pm}\right\rangle=0
\end{aligned}
$$

The explicit expressions for $S_{ \pm}^{\prime}$ in the exact classical solutions (2) have been constructed in ref. [9]. For $\left|x-z_{i}\right| \rightarrow \infty$ and $\left|y-z_{i}\right| \rightarrow \infty$, the propagation function $\left\langle x\left|S_{ \pm}^{\prime}\right| y\right\rangle$ approaches the free massless propagation function

$$
\left\langle x\left|S_{0}\right| y\right\rangle=\left\langle x\left|(\not)^{-1}\right| y\right\rangle .
$$

On the other hand, for $\left|x-z_{i}\right| \rightarrow \infty$ but $\left|y-z_{i}\right|$ finite, eq. (14a) indicates that

$$
\left\langle x\left|S_{ \pm}^{\prime}\right| y\right\rangle \rightarrow \mathrm{O}\left(\frac{1}{\left|x-z_{i}\right|^{2}}\right) \psi_{0 \pm}^{\dagger}\left(y-z_{i}\right)
$$

where we assumed the asymptotic behavior of the zero-mode wave function as given by eq. (9).

We now proceed to the construction of the fermion propagation function in the background gauge field (1). The gauge potential (1) has the total Pontryagin number $w=n_{+}-\left(n-n_{+}\right)$, and, according to the general theorem (7), the operator $\gamma_{\mu} D_{\mu}$ possesses at least $\left|n_{+}-\left(n-n_{+}\right)\right|$number of exact fermion zero modes. We again introduce a small fermion mass $m$ to regulate such vanishing eigenvalues and define the propagation function $S$ by

$$
\left(\gamma_{\mu} D_{\mu}+i m\right) S=\left[\gamma_{\mu}\left(\partial_{\mu}-i g \sum_{i=1}^{n} \boldsymbol{A}_{\mu i} \cdot \frac{1}{2} \boldsymbol{\tau}\right)+i m\right] S=I .
$$

For small $m$ and $1 /\left|z_{i}-z_{j}\right|(i \neq j, i, j=1,2, \ldots, n)$, we wish to express $S$ in terms of individual fermion zero modes $\left|\psi_{0 i}\right\rangle$ and fermion propagation functions $S_{i}^{\prime}$ defined by eq. (14). Here, we note that the operator $\gamma_{\mu} D_{\mu}+i m$ is non-hermitian, and we prefer dealing with a hermitian operator. We thus introduce

$$
\tilde{S}=-i \gamma_{5} S \text {, }
$$


then eq. (17) becomes

$$
\left(\tilde{\gamma}_{\mu} D_{\mu}-m \gamma_{5}\right) \tilde{S}=I,
$$

where we denoted $\tilde{\gamma}_{\mu}=-i \gamma_{5} \gamma_{\mu}=-\tilde{\gamma}_{\mu}^{\dagger}$. The operator $\tilde{\gamma}_{\mu} D_{\mu}-m \gamma_{5}$ is a hermitian operator and thus the inverse must be also hermitian, viz.

$$
\tilde{S}^{\dagger}=\tilde{S} \text {. }
$$

Since this hermiticity relation is very useful, we shall first construct $\tilde{S}$ as a double power series in $m$ and $1 / d$ (' $d$ ' characterizes the relative distances between instantons and anti-instantons), and then use the relation (18) to find $S$. Note that we do not make any assumptions about the magnitude of $m d$.

Let us write

$$
\tilde{S}=\tilde{S}_{0}+\sum_{i=1}^{n}\left(\tilde{S}_{i}^{\prime}-\tilde{S}_{0}\right)+\tilde{T}
$$

with

$$
\tilde{S}_{0}=-i \gamma_{5} S_{0}, \quad \tilde{S}_{i}^{\prime}=-i \gamma_{5} S_{i}^{\prime}
$$

From eq. (19) and the relation

$$
\left[\tilde{\gamma}_{\mu}\left(\partial_{\mu}-i g \boldsymbol{A}_{\mu i} \cdot \frac{1}{2} \tau\right)-m \gamma_{5}\right] \tilde{S}_{i}^{\prime}=I-\left|\psi_{0 i}\right\rangle\left\langle\psi_{0 i}\right|,
$$

we then obtain the following differential equation for $\tilde{T}$ :

$$
\left(\tilde{\gamma}_{\mu} D_{\mu}-m \gamma_{5}\right) \tilde{T}=\sum_{i=1}^{n}\left|\psi_{0 i}\right\rangle\left\langle\psi_{0 i}\right|+\tilde{R}
$$

with

$$
\tilde{R}=\sum_{i=1}^{n} i g \tilde{\gamma}_{\mu}\left(\boldsymbol{A}_{\mu} \cdot \frac{1}{2} \boldsymbol{\tau}-\boldsymbol{A}_{\mu i} \cdot \frac{1}{2} \tau\right)\left(\tilde{\boldsymbol{S}}_{i}^{\prime}-\tilde{\boldsymbol{S}}_{0}\right)+m \gamma_{5}\left[\tilde{\boldsymbol{S}}_{0}+\sum_{i=1}^{n}\left(\tilde{\boldsymbol{S}}_{i}^{\prime}-\tilde{\boldsymbol{S}}_{0}\right)\right]
$$

Note that the function $\tilde{R}$ is at most of order $1 / d^{2}$ or $m$. (Henceforth, we will use the notation $\mathrm{O}\left(m, 1 / d^{2}\right)$ to indicate this.) At this stage, we find it very convenient to introduce $\left|\tilde{Q}_{i}\right\rangle$ by

$$
\left(\tilde{\gamma}_{\mu} D_{\mu}-m \gamma_{5}\right)\left|\tilde{Q}_{i}\right\rangle=\left|\psi_{0 i}\right\rangle,
$$

assuming that $\left\langle x \mid \tilde{Q}_{i}\right\rangle$ 's are sufficiently bounded at infinity. Later, we will be able to express $\left|\tilde{Q}_{i}\right\rangle$ in terms of $\tilde{S}_{j}^{\prime}$ and $\left|\psi_{0_{j}}\right\rangle(j=1,2, \ldots, n)$. At this moment, it is sufficient to note that the functions $\left\langle x \mid \tilde{Q}_{i}\right\rangle$ are expected to involve large numbers like $1 / \mathrm{m}$ or $\left|z_{i}-z_{j}\right|^{3}$, since there is no bounded solution to eq. (26) with $m=0$ and the replacement

$$
\tilde{\gamma}_{\mu} D_{\mu} \rightarrow \tilde{\gamma}_{\mu}\left(\partial_{\mu}-i g \boldsymbol{A}_{\mu i} \cdot \frac{1}{2} \tau\right) .
$$

(If there should exist such a solution, we could have constructed a completely regular massless fermion propagation function in a single instanton potential.) 
As the starting approximation for $\tilde{T}$ (see eq. (24)), let us consider

$$
\tilde{T}^{(0)}=\sum_{i=1}^{n}\left|\tilde{Q}_{i}\right\rangle\left\langle\psi_{0 i}\right|
$$

Then, from eqs. (21) and (24), we obtain the integral equation

$$
\begin{aligned}
\tilde{S} & =\tilde{S}_{0}+\sum_{i=1}^{n}\left(\tilde{S}_{i}^{\prime}-\tilde{S}_{0}\right)+\tilde{T}^{(0)}+\tilde{\boldsymbol{S}} \tilde{R} \\
& \equiv \dot{\mathscr{S}}+\tilde{\mathscr{J}},
\end{aligned}
$$

where we defined $\tilde{\mathscr{I}}, \tilde{\mathscr{J}}$ by

$$
\begin{aligned}
& \dot{\mathscr{S}}=\tilde{S}_{0}+\sum_{i=1}^{n}\left(\tilde{S}_{i}^{\prime}+\tilde{S}_{0}\right)+\tilde{\mathscr{S}} \tilde{R}, \\
& \tilde{\mathscr{J}}=\sum_{i=1}^{n}\left|\tilde{Q}_{i}\right\rangle\left\langle\psi_{0 i}\right|+\tilde{\mathscr{J}} \tilde{R} .
\end{aligned}
$$

If we define $\left\langle\tilde{P}_{i}\right|$ by the integral equation

$$
\left\langle\tilde{P}_{i}\right|=\left\langle\psi_{0 i}\right|+\left\langle\tilde{P}_{i}\right| \tilde{R},
$$

the integral equation (31) takes the following simple form

$$
\tilde{\mathscr{E}}=\sum_{i=1}^{n}\left|\tilde{Q}_{i}\right\rangle\left\langle\tilde{P}_{i}\right|
$$

Note that eqs. (29)-(33) are still exact. Moreover, the integral equations (30) and (32) do not involve the yet unknown functions $\left\langle\tilde{Q}_{i}\right\rangle$, and thus, by iterating these integral equations, one may calculate $\tilde{\mathscr{S}}$ and $\tilde{\mathscr{J}}$ to any desired accuracy. To determine the functions $\left|\tilde{Q}_{i}\right\rangle$, we note that, from eq. (26),

$$
\begin{aligned}
\left|\tilde{Q}_{i}\right\rangle & =\tilde{S}\left|\psi_{0 i}\right\rangle \\
& =\tilde{\mathscr{S}}\left|\psi_{0 i}\right\rangle+\sum_{j=1}^{n}\left|\tilde{Q}_{j}\right\rangle\left\langle\tilde{P}_{j} \mid \tilde{\psi}_{0 i}\right\rangle,
\end{aligned}
$$

or

$$
\sum_{j=1}^{n}\left|\tilde{Q}_{i}\right\rangle\left(\delta_{j i}-\left\langle\tilde{P}_{j} \mid \tilde{\psi}_{0 i}\right\rangle\right)=\tilde{\mathscr{P}}\left|\psi_{0 i}\right\rangle
$$

In principle, eq. (35) allows one to determine $\left|\tilde{Q}_{j}\right\rangle$ to any desired accuracy. However, for the purpose of practical calculation, eq. (35) is not so convenient since one often has to deal with the ratios of very small numbers here. Actually, it is simpler to use the differential equation (26) directly (rather than its integrated form, eq. (24)) and the hermiticity relation (20). This will be explained below. 
From the hermiticity relation (20) and eq. (29), we conclude that

$$
\begin{aligned}
\tilde{\mathscr{J}}-\tilde{\mathscr{J}}^{\dagger} & =-\tilde{\mathscr{I}}+\tilde{\mathscr{S}}^{\dagger} \\
& =\mathrm{O}\left(m, \frac{1}{d^{2}}\right),
\end{aligned}
$$

where the estimate has been made from eq. (30). Using the representation (33) for $\tilde{\mathscr{I}}$, the relation $(36)$ now reads

$$
\sum_{i}^{n}\left\{\left|\tilde{Q}_{i}\right\rangle\left\langle\tilde{P}_{i}|-| \tilde{P}_{i}\right\rangle\left\langle\tilde{Q}_{i}\right|\right\}=\mathrm{O}\left(m, \frac{1}{d^{2}}\right) .
$$

Eq. (37) implies that

$$
\left|\tilde{Q}_{i}\right\rangle=\tilde{C}_{i j}\left|\tilde{P}_{j}\right\rangle+\mathrm{O}\left(m, \frac{1}{d^{2}}\right)
$$

with the constants $\tilde{C}_{i j}$ satisfying the hermiticity relation

$$
\tilde{C}_{i j}^{*}=\tilde{C}_{i i}
$$

The numerical values of the constants $\tilde{C}_{i j}$ may be determined by inserting the form (38) into the differential equation (26).

Beforc we look at the differential equation (26), let us first study $\left|\tilde{P}_{i}\right\rangle$, defined by eq. (32), in a little detail. Iterating eq. (32) once and using the explicit expression for $\tilde{R}$ (eq. (25)), we obtain

$$
\begin{aligned}
\left|\tilde{P}_{i}\right\rangle & =\left|\psi_{0 i}\right\rangle+\left\{\sum_{i=1}^{n}\left(\tilde{S}_{i}^{\prime}-\tilde{S}_{0}\right) i g \tilde{\gamma}_{\mu}\left(\boldsymbol{A}_{\mu} \cdot \frac{1}{2} \boldsymbol{\tau}-\boldsymbol{A}_{\mu i} \cdot \frac{1}{2} \boldsymbol{\tau}\right)\right. \\
& \left.+\left[\tilde{S}_{0}+\sum_{i=1}^{n}\left(\tilde{S}_{j}^{\prime}-\tilde{S}_{0}\right)\right] m \gamma_{5}\right\}\left|\psi_{0 i}\right\rangle+\mathrm{O}\left(m^{2}, m \frac{1}{d^{2}}, \frac{1}{d^{5}}\right),
\end{aligned}
$$

where we used the ket-notation instead of the bra-notation used in eq. (32). Further ignoring terms of $\mathrm{O}\left(1 / d^{5}\right)$ in eq. (40), we obtain

$$
\begin{aligned}
\left|\tilde{P}_{i}\right\rangle & =\left|\psi_{0 i}\right\rangle+\sum_{j \neq i}\left(\tilde{\boldsymbol{S}}_{i}^{\prime}-\tilde{\boldsymbol{S}}_{0}\right) i g \tilde{\gamma}_{\mu} \boldsymbol{A}_{\mu i} \cdot \frac{1}{2} \boldsymbol{\tau}\left|\psi_{0 i}\right\rangle \\
& +\left(\tilde{S}_{i}^{\prime}-\tilde{S}_{0}\right) i g \tilde{\gamma}_{\mu}\left(\boldsymbol{A}_{\mu} \cdot \frac{1}{2} \boldsymbol{\tau}-\boldsymbol{A}_{\mu i} \cdot \frac{1}{2} \boldsymbol{\tau}\right)\left|\psi_{0 i}\right\rangle \\
& +\left[\tilde{S}_{0}+\sum_{j=1}^{n}\left(\tilde{\boldsymbol{S}}_{i}^{\prime}-\tilde{\boldsymbol{S}}_{0}\right)\right] m \gamma_{5}\left|\psi_{0 i}\right\rangle+\mathrm{O}\left(m^{2}, m \frac{1}{d^{2}}, \frac{1}{d^{5}}\right)
\end{aligned}
$$

However, we now note that the second term in the right-hand side of eq. (41) can 
be written as

$$
\begin{aligned}
& \sum_{j \neq i}^{n}\left(\tilde{\boldsymbol{S}_{j}^{\prime}}-\tilde{S}_{0}\right) \tilde{\gamma}_{\mu} \partial_{\mu}\left|\psi_{0 i}\right\rangle \\
& \quad=-\sum_{j \neq i}^{n}\left|\psi_{0 j}\right\rangle\left\langle\psi_{0 j} \mid \psi_{0 i}\right\rangle+\sum_{i \neq i}^{n} \tilde{S}_{i}^{\prime} i g \tilde{\gamma}_{\mu} \boldsymbol{A}_{\mu j} \cdot \frac{1}{2} \tau\left|\psi_{0 i}\right\rangle .
\end{aligned}
$$

From eqs. (41) and (42), we now obtain

$$
\begin{aligned}
\left|\tilde{P}_{i}\right\rangle & =\left|\psi_{0 i}\right\rangle-\sum_{i \neq i}^{n}\left|\psi_{0 i}\right\rangle\left\langle\psi_{0 i} \mid \psi_{0 i}\right\rangle \\
& +\sum_{j \neq i}^{n}\left(\tilde{S}_{i}^{\prime}+\tilde{S}_{i}^{\prime}-\tilde{S}_{0}\right)\left(i g \tilde{\gamma}_{\mu} \boldsymbol{A}_{\mu i} \cdot \frac{1}{2} \boldsymbol{\tau}+m \gamma_{5}\right)\left|\psi_{0 i}\right\rangle \\
& +\mathrm{O}\left(m^{2}, m \frac{1}{d^{2}}, \frac{1}{d^{5}}\right)
\end{aligned}
$$

From eq. (43), it is a simple matter to obtain the result

$$
\begin{aligned}
& \left(\tilde{\gamma}_{\mu} D_{\mu}-m \gamma_{5}\right)\left|\tilde{P}_{i}\right\rangle=-m\left|\psi_{0 i}\right\rangle\left\langle\psi_{0 i}\left|\gamma_{5}\right| \psi_{0 i}\right\rangle \\
& \quad-\sum_{j \neq i}^{n}\left|\psi_{0 j}\right\rangle\left\langle\psi_{0 j}\left|\operatorname{ig} \tilde{\gamma}_{\mu} \boldsymbol{A}_{\mu j} \cdot \frac{1}{2} \boldsymbol{\tau}\right| \psi_{0 i}\right\rangle+\mathrm{O}\left(m^{2}, m \frac{1}{d^{2}}, \frac{1}{d^{5}}\right) .
\end{aligned}
$$

We have thus found that $\left(\tilde{\gamma}_{\mu} D_{\mu}-m \gamma_{5}\right)\left|\tilde{P}_{i}\right\rangle$ yields a linear combination of fermion zero modes. (For this, astute readers will realize that the first iteration term in eq. (40) is absolutely necessary.) This is precisely what is expected from eqs. (26) and (38). Now, let us define the constant matrices $\tilde{B}$ and $\tilde{C}$ by

$$
\begin{aligned}
& \{\tilde{B}\}_{i k}=\left\langle\psi_{0 k}\left|\left(\tilde{\gamma}_{\mu} D_{\mu}-m \gamma_{5}\right)\right| \tilde{P}_{i}\right\rangle, \\
& \{\tilde{C}\}_{i k}=C_{i k},
\end{aligned}
$$

then, from eqs. (26), (38) and (41), we obtain the matrix equation

$$
\tilde{C} \tilde{B}=I \text {. }
$$

If we denote

$$
\begin{aligned}
& \left\langle\psi_{0 i}\left|\gamma_{5}\right| \psi_{0 i}\right\rangle=-\delta_{i}, \\
& \quad\left(\delta_{i}=1 \text { for } i=1, \ldots, n_{+} \text {and } \delta_{i}=-1 \text { for } i=n_{+}+1, \ldots, n\right), \\
& \left\langle\psi_{0 j}\left|i g \tilde{\gamma}_{\mu} \boldsymbol{A}_{\mu j} \cdot \frac{1}{2} \boldsymbol{\tau}\right| \psi_{0 i}\right\rangle=\left\langle\psi_{0 j}\left|i g \tilde{\gamma}_{\mu} \boldsymbol{A}_{\mu i} \cdot \frac{1}{2} \tau\right| \psi_{0 i}\right\rangle=\tilde{a}_{j i},
\end{aligned}
$$

eq. (49) indicates that

$$
\{\tilde{B}\}_{i k}=m \delta_{i} \delta_{i k}-\tilde{a}_{k i}+\mathrm{O}\left(m^{2}, m \frac{1}{d^{2}}, \frac{1}{d^{5}}\right) .
$$


Since $\tilde{a}_{k i}^{*}=a_{i k}$, we have $\tilde{B}^{\dagger}=\tilde{B}$ and $\tilde{C}^{\dagger}=\tilde{C}$, up to the order being considered here. Moreover, from the asymptotic behavior (9) for zero-mode wave functions, we observe that, in general

$$
\tilde{a}_{i i}= \begin{cases}\mathrm{O}\left(\frac{1}{\left|z_{i}-z_{j}\right|^{3}}\right), & \text { if } w\left(A_{i}\right)=-w\left(A_{j}\right), \\ 0, & \text { if } w\left(A_{i}\right)=w\left(A_{j}\right) .\end{cases}
$$

Also, simply by checking chiralities (or $\gamma_{5}$-symmetry) in eqs. (32) and (45), one immediately concludes that

$$
\{\tilde{B}\}_{i k}=m\left\{\delta_{i} \delta_{i k}+\mathrm{O}\left(m, 1 / d^{2}\right)\right\}, \quad \text { if } w\left(A_{i}\right)=w\left(A_{j}\right) .
$$

From these behaviors, it is clear that the constraints $\tilde{C}_{i j}$ are in general of order $1 / \mathrm{m}$ or $d^{3}$.

Now, we thus conclude that the functions $\left|\tilde{Q}_{i}\right\rangle$ are given by

$$
\left|\tilde{Q}_{i}\right\rangle=\left|\tilde{Q}_{i}^{(0)}\right\rangle+\left|\tilde{\delta_{i}}\right\rangle
$$

with

$$
\begin{aligned}
& \left|\tilde{Q}_{i}^{(0)}\right\rangle=\tilde{C}_{i j}\left\{\left|\psi_{0 j}\right\rangle+\left[\sum_{k=1}^{n}\left(\tilde{\boldsymbol{S}}_{k}^{\prime}-\tilde{\boldsymbol{S}}_{0}\right) i g \tilde{\gamma}_{\mu}\left(\boldsymbol{A}_{\mu} \cdot \frac{1}{2} \boldsymbol{\tau}-\boldsymbol{A}_{\mu k} \cdot \frac{1}{\tilde{2}} \boldsymbol{\tau}\right)\right.\right. \\
& \left.\left.\quad+\left[\tilde{\boldsymbol{S}}_{0}+\sum_{k=1}^{n}\left(\tilde{\boldsymbol{S}}_{k}^{\prime}-\tilde{\boldsymbol{S}}_{0}\right)\right] m \gamma_{5}\right]\right\}\left|\psi_{0 j}\right\rangle \\
& \left|\tilde{\delta}_{i}\right\rangle=\mathrm{O}\left(m, \frac{1}{d^{2}}\right)
\end{aligned}
$$

Due to eqs. (26), (38), (44) and (47), the correction $\left|\tilde{\delta}_{i}\right\rangle$ in eq. (53) obeys

$$
\begin{aligned}
\left(\tilde{\gamma}_{\mu} D_{\mu}-m \gamma_{5}\right)\left|\tilde{\delta}_{i}\right\rangle & =\left|\psi_{0 i}\right\rangle-\left(\tilde{\gamma}_{\mu} D_{\mu}-m \gamma_{5}\right)\left|\tilde{Q}_{i}^{(0)}\right\rangle \\
& =\mathrm{O}\left(m^{2}, m \frac{1}{d^{2}}, \frac{1}{d^{5}}\right) .
\end{aligned}
$$

If we denote

$$
\left|\tilde{\varepsilon}_{i}\right\rangle=\left|\psi_{0 i}\right\rangle-\left(\tilde{\gamma}_{\mu} D_{\mu}-m \gamma_{5}\right)\left|\tilde{Q}_{i}^{(0)}\right\rangle
$$

we now obtain the integral equation

$$
\begin{aligned}
\left|\tilde{Q}_{i}\right\rangle & =\left|\tilde{Q}_{i}^{(0)}\right\rangle+\tilde{S}\left|\tilde{\varepsilon}_{i}\right\rangle \\
& =\left|\tilde{Q}_{i}^{(0)}\right\rangle+\tilde{\mathscr{S}}\left|\tilde{\varepsilon}_{i}\right\rangle+\sum_{i=1}^{n}\left|\tilde{Q}_{j}\right\rangle\left\langle\tilde{P}_{j} \mid \tilde{\varepsilon}_{i}\right\rangle
\end{aligned}
$$

Eqs. (29), (30), (32), (33) and (58) form a complete set of integral equations which allow one to calculate $\tilde{S}$ to an arbitrary accuracy. 
It is a simple matter to translate our findings for $\tilde{S}$ into those for $S$ via eq. (18). The results may be summarized by the following equations:

$$
\begin{aligned}
& S=\mathscr{S}+\mathscr{J}, \\
& \mathscr{S}=S_{0}+\sum_{i=1}^{n}\left(S_{i}^{\prime}-S_{0}\right)+\mathscr{S R}(m) \\
& \mathscr{J}=\sum_{i=1}^{n}\left|Q_{i}\right\rangle\left\langle P_{i}\right| \\
& R(m)=\sum_{i=1}^{n} i g \gamma_{\mu}\left(\boldsymbol{A}_{\mu} \cdot \frac{1}{2} \boldsymbol{\tau}-\boldsymbol{A}_{\mu i} \cdot \frac{1}{2} \tau\right)\left(\boldsymbol{S}_{i}^{\prime}-S_{0}\right) \\
& \quad-i m\left[S_{0}+\sum_{i=1}^{n}\left(S_{i}^{\prime}-S_{0}\right)\right] \\
& \left\langle P_{i}\right|=\left\langle\psi_{0 i}\right|+\left\langle P_{i}\right| R(m) \\
& \quad=\left\langle\psi_{0 i}\right|+\left\langle\psi_{0 i}\right| R(m)+\mathrm{O}\left(m^{2}, m \frac{1}{d^{2}}, \frac{1}{d^{5}}\right) \\
& \left|Q_{i}\right\rangle=\left|Q_{i}^{(0)}\right\rangle+\mathscr{S}\left|\varepsilon_{i}\right\rangle+\sum_{i=1}^{n}\left|Q_{j}\right\rangle\left\langle P_{j} \mid \varepsilon_{i}\right\rangle \\
& \left.\left|Q_{i}^{(0)}\right\rangle=C_{i j}\left\{\left|\psi_{0 j}\right\rangle+R^{\dagger}(-m) \mid \psi_{0 j}\right)\right\} \\
& C B=I, \\
& \{C\}_{i k}=C_{i k}, \quad\{B\}_{i k}=i m \delta_{i k}+a_{k i}, \\
& a_{k i}=a_{i k}^{*}=\left\langle\psi_{0 k}\left|-i g \gamma_{\mu} \boldsymbol{A}_{\mu k} \cdot \frac{1}{2} \tau\right| \psi_{0 i}\right\rangle \\
& \left|\varepsilon_{i}\right\rangle=\left|\psi_{0 i}\right\rangle-\left(\gamma_{\mu} D_{\mu}+i m\right)\left|Q_{i}^{(0)}\right\rangle, \quad\left(=\mathrm{O}\left(m^{2}, m \frac{1}{d^{2}}, \frac{1}{d^{5}}\right)\right)
\end{aligned}
$$

In leading order, the fermion propagation function $S$ is given by

$$
\begin{aligned}
S= & S_{0}+\sum_{i=1}^{n}\left(S_{i}^{\prime}-S_{0}\right)+C_{i j}\left\{\left|\psi_{0 j}\right\rangle+R^{\dagger}(-m)\left|\psi_{0 i}\right\rangle\right\} \\
& \times\left\{\left\langle\psi_{0 i}\right|+\left\langle\psi_{0 i}\right| R(m)\right\}+\mathrm{O}\left(m, \frac{1}{d^{2}}\right) .
\end{aligned}
$$

(Precisely speaking, the expression (60) for $\langle x|S| y\rangle$ is valid only for $|x-y| \ll m^{-1}$. This is because a constant mass term in the Green function equation does not damp asymptotically. However, this can be easily fixed by keeping the asymptotic mass tail of a free particle when we take the zero-mass limit in eq. (11) and then repeating the above construction.) 
With the exact classical solutions (2) for $A_{\mu i}^{a}$, a simple calculation using the zero-mode wave functions (5) yields

$$
a_{k i}=-i \frac{4 \rho_{k} \rho_{i}}{\left|z_{k}-z_{i}\right|^{4}}\left[\left(z_{k}-z_{i}\right) \cdot q_{k i}\right]\left[1+\mathrm{O}\left(\frac{1}{\left|z_{k}-z_{i}\right|^{2}}\right)\right],
$$

for $w\left(\boldsymbol{A}_{i}\right)=1=-w\left(\boldsymbol{A}_{k}\right)$, where $\left(q_{k i}\right)_{\mu}$ is a real four-vector specifying the relative global gauge orientation according to

$$
\begin{aligned}
& \mathrm{e}^{i \boldsymbol{\omega}_{k i} \cdot \tau / 2}=\mathrm{e}^{i \boldsymbol{\Omega}_{k} \cdot \tau / 2} \mathrm{e}^{-i \boldsymbol{\Omega}_{i} \cdot \tau / 2}, \\
& \left(q_{k i}\right)_{\mu}=\left(\hat{\omega}_{k i} \sin \frac{1}{2}\left|\boldsymbol{\omega}_{k i}\right|, \cos \frac{1}{2}\left|\boldsymbol{\omega}_{k i}\right|\right) .
\end{aligned}
$$

For a specific relative global gauge orientation satisfying

$$
\left(z_{k}-z_{i}\right) \cdot q_{k i}=0
$$

the constants $C_{i j}$ involve terms of $0\left(\left|z_{k}-z_{i}\right|^{5}\right)$, and one must include the second (or morc) itcration for $\left|P_{i}\right\rangle$ in eq. (40) before onc determines $\left|\tilde{Q}_{i}\right\rangle$ by cqs. (38) and (26). We have not done this calculation yet, and the physical significance of such specific relative gauge orientations are not clear. If all the higher-order terms also vanish, that implies the presence of an exact fermion zero mode. Thus, we may say that, if a certain widely separated instanton-anti-instanton system (i.e., $n_{+}=n-n_{+}=1$ ) might possess an exact fermion zero mode, the relative global gauge orientation between the instanton and anti-instanton should obey eq. (63).

In the limit $m \gg d^{-3}$, the expression (60) is reduced to

$$
S=S_{0}+\sum_{i=1}^{n}\left[S_{i}^{\prime}+\frac{\left|\psi_{0 i}\right\rangle\left\langle\psi_{0 i}\right|}{i m}-S_{0}\right]
$$

which is the expression used in the dilute-gas approximation. For $m \leqslant d^{-3}$, it depends crucially on what kind of background gauge fields are being considered. If the gauge field $A_{i}^{a}$ includes only instantons (or anti-instantons only), then $a_{k i} \equiv 0$ and the expression (64) still provides a good approximation. On the other hand, if $n_{+} \neq 0$ or $n$, one has to solve the full matrix equation $(59 \mathrm{~h})$ and keep the full expression (60). For example, for $n_{+}=n-n_{+}=1$ (i.e., an instanton and antiinstanton), we obtain

$$
\begin{aligned}
S= & S_{1}^{\prime}+S_{2}^{\prime}-S_{0}-\frac{i m}{m^{2}+|a|^{2}} \\
& \times\left\{\left|\psi_{01}\right\rangle+R^{\dagger}(-m)\left|\psi_{01}\right\rangle\right\}\left\{\left\langle\psi_{01}\right|+\left\langle\psi_{01}\right| R(m)\right\} \\
& \left.-\frac{i m}{m^{2}+|a|^{2}}\left\{\left|\psi_{02}\right\rangle+R^{\dagger}(-m) \mid \psi_{02}\right)\right\}\left\{\left\langle\psi_{02}\right|+\left\langle\psi_{02}\right| R(m)\right\} \\
& +\frac{a^{*}}{m^{2}+|a|^{2}}\left\{\left|\psi_{01}\right\rangle+R^{\dagger}(-m)\left|\psi_{01}\right\rangle\right\}\left\{\left\langle\psi_{02}\right|+\left\langle\psi_{02}\right| R(m)\right\}
\end{aligned}
$$




$$
+\frac{a}{m^{2}+|a|^{2}}\left\{\left|\psi_{02}\right\rangle+R^{+}(-m)\left|\psi_{02}\right\rangle\right\}\left\{\left\langle\psi_{01}\right|+\left\langle\psi_{01}\right| R(m)\right\}+\mathrm{O}\left(m, \frac{1}{\left|z_{1}-z_{2}\right|^{2}}\right),
$$

where the constant $a$ is given by

$$
a=\left\langle\psi_{02}\left|-i g \gamma_{\mu} \boldsymbol{A}_{\mu 2} \cdot \frac{1}{2} \tau\right| \psi_{01}\right\rangle .
$$

Note that, in the limit $m \ll 1 /\left|z_{1}-z_{2}\right|^{3}$ (or, more precisely, $m \ll|a|$ ), the expression (65) approaches the following $m$-independent expression

$$
\begin{aligned}
S= & S_{1}^{\prime}+S_{2}^{\prime}-S_{0}+a^{-1}\left\{\left|\psi_{01}\right\rangle+R^{\dagger}(m=0)\left|\psi_{01}\right\rangle\right\} \\
& \times\left\{\left\langle\psi_{02}\left|+\left\langle\psi_{02}\right| R(m=0)\right\}+\left(a^{*}\right)^{-1}\left\{\left|\psi_{02}\right\rangle+R^{\dagger}(m=0)\left|\psi_{02}\right\rangle\right\}\right.\right. \\
& \times\left\{\left\langle\psi_{01}\right|+\left\langle\psi_{01}\right| R(m=0)\right\}+\mathrm{O}\left(\frac{1}{\left|z_{1}-z_{2}\right|^{2}}\right) .
\end{aligned}
$$

Here, we note that the terms like $R^{\dagger}(m=0)\left|\psi_{01}\right\rangle\left\langle\psi_{02}\right|$ in eq. (67) represent interference terms between the zero modes and non-normalizable continuum, and in general are of the same order with, say, $\left|\psi_{01}\right\rangle\left\langle\psi_{02}\right|$. For example, when $x$ any $y$ are close to $z_{2}$, both $\left\langle x\left|R^{+}(-m)\right| \psi_{01}\right\rangle\left\langle\psi_{2} \mid y\right\rangle$ and $\left\langle x \mid \psi_{01}\right\rangle\left\langle\psi_{02} \mid y\right\rangle$ are $0\left(1 /\left(\left|z_{1}-z_{2}\right|^{3}\right)\right)$. For a background gauge field with $n_{+} \neq n-n_{+}$, even in the limit $m \ll 1 / d^{3}$ the propagation function involves a singular term proportional to $1 / m$ as well as $m$ independent terms as shown in eq. (67). Of course, this implies the presence of exact normalizable zero modes for the massless Dirac equation, in accordance with the general theorem (7).

The fermion propagation function $\langle x|S| y\rangle$, given in eq. (60), is particularly simple when the space-time points $x$ and $y$ are not close to a certain (anti-) instanton simultaneously. Namely, if $\left|x-z_{i}\right|$ and $\left|y-z_{i}\right|$ are not simultaneously of order of (or smaller than) the $i$ th (anti-) instanton size for any $i$, the expression (60) may be approximated by

$$
S_{0}+C_{i j}\left|\psi_{0 j}\right\rangle\left\langle\psi_{0 i}\right| \text {. }
$$

The second term here is precisely what one would expect from the so-called zeromode approximation $[2,13]$; i.e., restrict Hilbert space to zero-mode subspaces and find the inverse of the operator

$$
\left|\psi_{0 i}\right\rangle\left\langle\psi_{0 i}\left|\gamma_{\mu} D_{\mu}+i m\right| \psi_{0 k}\right\rangle\left\langle\psi_{0 k}\right| \text {. }
$$

However, in a background gauge field which includes both instantons and antiinstantons, the zero-mode approximation (68) for $\langle x|S| y\rangle$ is a bad approximation when $x, y$ are close to a single (anti-) instanton simultaneously. Thus, to calculate quantities such as vacuum polarization currents (which involve the propagation function at $x=y$ ), one must use the full expression (60). Here, we note that the zero-mode approximation is a priori not justified in a gauge field including both instantons and anti-instantons. For example, in the instanton-anti-instanton system, 
absence of an exact normalizable zero mode immediately implies that we have only a non-normalizable continuum ( $\not D D$ is a positive semidefinite operator). This simple fact indicates that, if one wishes to use the degenerate perturbation theory, one must do so with two normalizable zero modes and the non-normalizable continuum [13]. Acute readers will realize that we have effectively solved this seemingly very delicate problem with a judicious use of the propagation function in the non-zero-mode subspace. Finally, we note that, using the fermion propagation function (60) with appropriate regularization of short-distance divergences (for example, see ref. [11] and also sect. 3 for the isospin current), one may explicitly verify the following divergence relations:

$$
\begin{aligned}
\partial_{\mu}\left\langle J_{\mu}(x)\right\rangle & =-i \partial_{\mu} \operatorname{Tr}\left(\gamma_{\mu}\langle x|S| x\rangle\right)=0, \\
D_{\mu}^{a b}\left(J_{\mu}^{b}(x)\right\rangle & =-i\left(\partial_{\mu} \delta_{a b}+g \epsilon_{a c b} A_{\mu}^{c}(x)\right) \operatorname{Tr}\left(\gamma_{\mu} \frac{1}{2} \tau^{b}\langle x|S| x\rangle\right)=0, \\
\partial_{\mu}\left\langle J_{\mu}^{5}(x)\right\rangle & =\partial_{\mu} \operatorname{Tr}\left(\gamma_{S} \gamma_{\mu}\langle x|S| x\rangle\right) \\
& =-2 i m \operatorname{Tr}\left(\gamma_{5}\langle x|S| x\rangle\right)-\frac{g^{2}}{16 \pi^{2}} F_{\mu \nu}^{a}(x)^{*} F_{\mu \nu}^{a}(x) .
\end{aligned}
$$

up to $0\left(m, 1 / d^{2}\right)$. We leave this exercise to interested readers.

\section{Fermionic effective action}

Using the propagation function constructed in sect. 2, we now calculate the fermionic effect action. The fermionic effective action $\Gamma_{\mathrm{f}}(A)$ is defined by

$$
\mathrm{e}^{\Gamma_{f}(\boldsymbol{A})}=\frac{\operatorname{det}\left[-i \gamma_{\mu}\left(\partial_{\mu}-i g \boldsymbol{A}_{\mu} \cdot \frac{1}{2} \boldsymbol{\tau}\right)+m\right]}{\operatorname{det}\left[-i \gamma_{\mu} \partial_{\mu}+m\right]} .
$$

Using the variational formula

$$
\delta \ln \operatorname{det} X=\operatorname{Tr}\left(X^{-1} \delta X\right),
$$

we obtain $\left(N_{\mathrm{f}}\right.$ : number of fermion flavours)

$$
\delta \Gamma_{\mathrm{f}}(A)=-g N_{\mathrm{f}} \int \mathrm{d}^{4} x\left\langle J_{\mu}^{a}(x)\right\rangle \delta A_{\mu}^{a}(x),
$$

where the isospin current $\left\langle J_{\mu}^{\mathfrak{a}}(x)\right\rangle$ due to fermion vacuum polarization is given by

$$
\left\langle J_{\mu}^{a}(x)\right\rangle=i \operatorname{Tr}\left(\gamma_{\mu} \frac{1}{2} \tau^{a}\langle x|S| x\rangle\right) .
$$

The isospin current (74) is actually infinite, and requires a careful regularization and renormalization. The simplest regularization procedure is that of Pauli-Villars; i.e., introduce $N_{\mathrm{f}}$ number of regulator Fermi fields with very heavy mass $M$ and coupled to gauge fields according to a minimal gauge-invariant coupling. Then we 
may define the regularized isospin current such as

$$
\left\langle J_{\mu}^{a}(x)\right\rangle_{\mathrm{R}}=i \lim _{y \rightarrow x} \operatorname{Tr}\left\{\gamma_{\mu} \frac{1}{2} \tau\left[\langle x|S| y\rangle-\left\langle x\left|S_{\mathrm{R}}\right| y\right\rangle\right]\right\},
$$

where the regulator propagation function $S_{\mathrm{R}}$ satisfies

$$
\left(\gamma_{\mu} D_{\mu}+i M\right) S_{\mathrm{R}}=I \text {. }
$$

It is a simple matter to show that the expression (75) is logarithmically divergent as $M \rightarrow \infty$, being proportional to

$$
-\frac{2}{3} \frac{1}{8 \pi^{2}}(\log M) D_{\nu}^{u b} F_{\mu \nu}^{b}(x)
$$

This is a standard renormalization problem and the infinity (77) is absorbed into the coupling constant renormalization. Thus, we may define the renormalized isospin current by

$$
\begin{aligned}
& \left\langle J_{\mu}^{a}(x)\right\rangle_{\mathrm{ren}}=\lim _{M \rightarrow \infty}\left[i \lim _{y \rightarrow x} \operatorname{Tr}\left\{\gamma_{\mu} \frac{1}{2} \tau^{a}\left(\langle x|S| y\rangle-\left\langle x\left|S_{\mathbf{R}}\right| y\right\rangle\right)\right\}\right. \\
& \left.\quad+\frac{2}{3} \frac{1}{8 \pi^{2}} \log \frac{M}{\mu_{0}} D_{\nu}^{a b} F_{\mu \nu}^{b}(x)\right],
\end{aligned}
$$

where $\mu_{0}$ is the renormalization mass necessary to fix the coupling constant. Now the renormalized effective action satisfies the variational formula

$$
\delta \Gamma_{\mathrm{f}}(A)_{\text {ren }}=-g N_{\mathrm{f}} \int \mathrm{d}^{4} x\left\langle J_{\mu}^{a}(x)\right\rangle_{\text {ren }} \delta A_{\mu}^{a}(x) .
$$

For the background gauge potential (1), $D_{\nu}^{a b} F_{\mu \nu}^{b}(x)$ is strongly localized around individual (anti-)instantons, and thus, up to $\mathrm{O}\left(1 / d^{2}\right)$, the renormalization counter term in eq. (78) simply renormalizes the individual vacuum polarization currents due to non-zero modes. From the propagation function (60), we thus obtain the renormalized isospin current

$$
\begin{aligned}
& \left\langle J_{\mu}^{a}(x)\right\rangle_{\mathrm{ren}}=\sum_{i=1}^{n}\left\langle J_{\mu i}^{a}(x)\right\rangle_{\mathrm{ren}}^{\prime}+i C_{i j}\left\{\left\langle\psi_{0 i} \mid x\right\rangle+\left\langle\psi_{0 i}|R(m)| x\right\rangle\right\} \\
& \times \gamma_{\mu} \frac{1}{2} \tau^{a}\left\{\left\langle x \mid \psi_{0 j}\right\rangle+\left\langle x\left|R^{\dagger}(-m)\right| \psi_{0 j}\right\rangle\right\}+\mathrm{O}\left(m, \frac{1}{d^{2}}\right)
\end{aligned}
$$

with

$$
\begin{aligned}
& \left\langle J_{\mu i}^{a}(x)\right\rangle_{\mathrm{ren}}^{\prime}=\lim _{M \rightarrow \infty}\left[i \operatorname { l i m } _ { y \rightarrow x } \operatorname { T r } \left\{\gamma_{\mu} \frac{1}{2} \tau^{a}\left(\left\langle x\left|S_{i}^{\prime}\right| y\right\rangle-\left\langle x\left|S_{i \mathbf{R}}\right| y\right\rangle\right)\right.\right. \\
& \left.\quad+\frac{2}{3} \frac{1}{8 \pi^{2}} \log \frac{M}{\mu} D_{\nu i}^{a b} F_{\mu \nu i}^{b}(x)\right]
\end{aligned}
$$


where we denoted $F_{\mu \nu i}^{a}(x)=\partial_{\mu} A_{\nu i}^{a}-\partial_{\nu} A_{\mu i}^{a}+g \epsilon_{a b c} A_{\mu i}^{b} A_{\mu i}^{c}$. The exact expressions for $\left\langle J_{\mu}^{a}(x)\right\rangle_{\text {ren }}$ in the exact classical solutions (2) (i.e., $\left.D_{\nu}^{a b} F_{\mu \nu}^{b}(x)=0\right)$ have been calculated in ref. [11] with the result

$$
\left\langle J_{\mu \perp}^{a}(x)\right\rangle_{\operatorname{ren}}^{\prime} \frac{1}{2} \tau^{a}=\mathrm{e}^{-i \boldsymbol{\Omega} \cdot \tau / 2}\left(-\frac{1}{3 \pi^{2}}\right) \frac{\rho^{2} \eta_{\mu \nu a}^{(\mp)}(x-z)_{\nu} \frac{1}{2} \tau^{a}}{\left[(x-z)^{2}+\rho^{2}\right]^{3}} \mathrm{e}^{i \boldsymbol{\Omega} \cdot \tau / 2}
$$

We will assume that the currents $\left\langle J_{\mu i}^{a}(x)\right\rangle^{\prime}$ are strongly localized around $x=z_{i}$, as the expression (82) in the exact classical solution indicates. Note that the isospin current given in eq. (80) is then strongly localized around $x=z_{i}$ 's.

We now look at the variational formula (79) with the position variation of the ith (anti-) instanton

$$
\begin{aligned}
\delta A_{\mu}^{a}(x) & =\delta z_{i \nu} \frac{\partial}{\partial z_{i \nu}} A_{\mu}^{a}(x) \\
& =-\delta z_{i \nu} \frac{\partial}{\partial x_{\nu}} A_{\mu \nu}^{a}\left(x-z_{i}\right) \\
& =\delta z_{i \nu}\left[F_{\mu \nu i}^{a}\left(x-z_{i}\right)-\left(\partial_{\mu} \delta_{a b}+g \epsilon_{a c b} A_{\mu i}^{c}\left(x-z_{i}\right)\right) A_{\nu i}^{b}\left(x-z_{i}\right)\right] .
\end{aligned}
$$

Inserting the isospin current (80) and eq. (83) into the variational formula (79) and using the conservation law $(70 \mathrm{~b})$, we obtain

$$
\begin{aligned}
& \frac{\delta \Gamma_{\mathrm{f}}(A)_{\mathrm{ren}}}{\delta z_{i \nu}}=-g N_{\mathrm{f}} \int \mathrm{d}^{4} x\left[\left\langle J_{\mu i}^{a}(x)\right\rangle_{\mathrm{ren}}^{\prime}+i C_{i i}\left\{\left\langle\psi_{0 i} \mid x\right\rangle+\left\langle\psi_{0 i}|R(m)| x\right\rangle\right\}\right. \\
& \quad \times \gamma_{\mu} \frac{1}{2} \gamma^{a}\left\{\left\langle x \mid \psi_{0 i}\right\rangle+\left\langle x\left|R^{\dagger}(-m)\right| \psi_{0 i}\right\rangle\right\} \\
& \quad+i \sum_{j \neq i}^{n} C_{i j}\left\{\left\langle\psi_{0 i} \mid x\right\rangle+\left\langle\psi_{0 i}|R(m)| x\right\rangle\right\} \gamma_{\mu} \frac{1}{2} \tau^{a}\left\{\left\langle x \mid \psi_{0 j}\right\rangle+\left\langle x\left|R^{\dagger}(-m)\right| \psi_{0 j}\right\rangle\right\} \\
& \quad+i \sum_{i \neq i}^{n} C_{i i}\left\{\left\langle\psi_{0 j} \mid x\right\rangle+\left\langle\psi_{0 j}|R(m)| x\right\rangle\right\} \\
& \left.\quad \times \gamma_{\mu} \frac{1}{2} \tau^{a}\left\{\left\langle x \mid \psi_{0 i}\right\rangle+\left\langle x\left|R^{\dagger}(-m)\right| \psi_{0 i}\right\rangle\right\}\right] F_{\mu \nu i}^{a}\left(x-z_{i}\right)+\mathrm{O}\left(m, \frac{1}{d^{2}}\right)
\end{aligned}
$$

We now note that, since the self-action is translation-invariant, we have

$$
\int \mathrm{d}^{4} x\left\langle J_{\mu i}^{a}(x)\right\rangle_{\mathrm{ren}}^{\prime} F_{\mu \nu i}^{a}\left(x-z_{i}\right)=0
$$

Also, we note that

$$
\begin{aligned}
& \left\{\left\langle\psi_{0 i} \mid x\right\rangle+\left\langle\psi_{0 i}|R(m)| x\right\rangle\right\} \gamma_{\mu} \frac{1}{2} \tau^{a}\left\{\left\langle x \mid \psi_{0 i}\right\rangle+\left\langle x\left|R^{\dagger}(-m)\right| \psi_{0 i}\right)\right\} \\
& \quad=-i m \int \mathrm{d}^{4} y \psi_{0 i}^{\dagger}\left(x-z_{i}\right) \gamma_{\mu} \frac{1}{2} \tau^{a}\left[S_{0}(x, y)+\sum_{i=1}^{n}\left(S_{i}^{\prime}(x, y)-S_{0}(x, y)\right)\right] \psi_{0 i}\left(y-z_{i}\right)
\end{aligned}
$$




$$
\begin{aligned}
& -i m \int \mathrm{d}^{4} y \psi_{0 i}^{\dagger}\left(y-z_{i}\right)\left[S_{0}(y, x)+\sum_{i=1}^{n}\left(S_{i}^{\prime}(y, x)-S_{0}(y, x)\right)\right] \gamma_{\mu} \frac{1}{2} \tau^{a} \psi_{0 i}\left(x-z_{i}\right) \\
= & \mathrm{O}\left(m, \frac{1}{d^{2}}\right)
\end{aligned}
$$

and thus the term proportional to $C_{i i}$ in eq. (84) may be safely ignored. Thus, ignoring terms of $\mathrm{O}\left(\mathrm{m}, 1 / \mathrm{d}^{2}\right)$ or higher, eq. (84) may be simplified to

$$
\begin{aligned}
& \frac{\delta \Gamma_{\mathrm{f}}(A)_{\mathrm{ren}}}{\delta z_{i \nu}}=-i g N_{\mathrm{f}} \sum_{j \neq i} C_{i j}\left\{\left\langle\psi_{0 i}\left|\gamma_{\mu} \boldsymbol{F}_{\mu \nu i} \cdot \frac{1}{2} \tau\right| \psi_{0 j}\right\rangle\right. \\
& \left.\quad+\left\langle\psi_{0 i}\left|\gamma_{\mu} \boldsymbol{F}_{\mu \nu i} \cdot \frac{1}{2} \tau R^{\dagger}(-m)\right| \psi_{0 j}\right\rangle\right\} \\
& \quad-i g N_{\mathrm{f}} \sum_{j \neq i} C_{j i}\left\{\left\langle\psi_{0 j}\left|\gamma_{\mu} \boldsymbol{F}_{\mu \nu i} \cdot \frac{1}{2} \tau\right| \psi_{0 i}\right\rangle\right. \\
& \left.\quad+\left\langle\psi_{0 j}\left|R(m) \gamma_{\mu} \boldsymbol{F}_{\mu \nu i} \cdot \frac{1}{2} \tau\right| \psi_{0 i}\right\rangle\right\}+\mathrm{O}\left(m, \frac{1}{d^{2}}\right)
\end{aligned}
$$

Using the explicit expression (59d) for $R^{+}(-m)$, we now note that

$$
\begin{aligned}
I_{\nu, i j} & \equiv g\left\langle\psi_{0 i}\left|\gamma_{\mu} \boldsymbol{F}_{\mu \nu i} \cdot \frac{1}{2} \boldsymbol{\tau}\right| \psi_{0 j}\right\rangle+g\left\langle\psi_{0 i}\left|\gamma_{\mu} \boldsymbol{F}_{\mu \nu i} \cdot \frac{1}{2} \tau R^{\dagger}(-m)\right| \psi_{0 j}\right\rangle \\
& =g\left\langle\psi_{0 i}\left|\gamma_{\mu} \boldsymbol{F}_{\mu \nu i} \cdot \frac{1}{2} \boldsymbol{\tau}\left\{I+S_{i}^{\prime} i g \gamma_{\Lambda} \boldsymbol{A}_{\lambda i} \cdot \frac{1}{2} \boldsymbol{\tau}\right\}\right| \psi_{0 j}\right\rangle+\mathrm{O}\left(m \frac{1}{d^{2}}, \frac{1}{d^{5}}\right),
\end{aligned}
$$

where we performed some simple manipulations analogous to the ones used in eq. (42). Writing

$$
g \boldsymbol{F}_{\mu \nu i} \cdot \frac{1}{2} \boldsymbol{\tau}=i\left[D_{\mu i}, D_{\nu i}\right], \quad\left(D_{\mu i}=\partial_{\mu}-i g \boldsymbol{A}_{\mu i} \cdot \frac{1}{2} \boldsymbol{\tau}\right),
$$

the expression (88) may be also written in the forms

$$
\begin{aligned}
I_{\nu, i j} & =-i\left\langle\psi_{0 i}\left|D_{\nu i} D_{i}\right| \psi_{0 j}\right\rangle \\
& +i\left\langle\psi_{0 i}\right| D_{\nu i}\left[I-\left|\psi_{0 i}\right\rangle\left\langle\psi_{0 i}\left|i g \gamma_{\lambda} \boldsymbol{A}_{\lambda i} \cdot \frac{1}{2} \tau\right| \psi_{0 j}\right\rangle+\mathrm{O}\left(m \frac{1}{d^{2}}, \frac{1}{d^{5}}\right)\right. \\
= & -i\left\langle\psi_{0 i}\left|D_{\nu i} i g \gamma_{\lambda} \boldsymbol{A}_{\lambda j} \cdot \frac{1}{2} \boldsymbol{\tau}\right| \psi_{0 j}\right\rangle \\
& +i\left\langle\psi_{0 i}\left|D_{\nu i}\right| \psi_{0 i}\right\rangle\left\langle\psi_{0 i}\left|i g \gamma_{\lambda} \boldsymbol{A}_{\lambda i} \cdot \frac{1}{2} \tau\right| \psi_{0 j}\right\rangle+\mathrm{O}\left(m \frac{1}{d^{2}}, \frac{1}{d^{5}}\right) \\
= & -i \frac{\partial}{\partial z_{\nu i}}\left\langle\psi_{0 i}\left|i g \gamma_{\lambda} \boldsymbol{A}_{\lambda i} \cdot \frac{1}{2} \tau\right| \psi_{0 j}\right\rangle \\
& +i\left\langle\psi_{0 i}\left|D_{\nu i}\right| \psi_{0 i}\right\rangle\left\langle\psi_{0 i}\left|i g \gamma_{\lambda} \boldsymbol{A}_{\lambda i} \cdot \frac{1}{2} \tau\right| \psi_{0 j}\right\rangle+\mathrm{O}\left(m \frac{1}{d^{2}}, \frac{1}{d^{5}}\right) .
\end{aligned}
$$


Similarly, we obtain

$$
\begin{aligned}
& g\left\langle\psi_{0 j}\left|\gamma_{\mu} \boldsymbol{F}_{\mu \nu i} \cdot \frac{1}{2} \boldsymbol{\tau}\right| \psi_{0 i}\right\rangle+g\left\langle\psi_{0 j}\left|R(m) \gamma_{\mu} \boldsymbol{F}_{\mu \nu t} \cdot \frac{1}{2} \boldsymbol{\tau}\right| \psi_{0 i}\right\rangle \\
& =-i \frac{\partial}{\partial z_{\nu i}}\left\langle\psi_{0 j}\left|i g \gamma_{\lambda} \boldsymbol{A}_{\lambda j} \cdot \frac{1}{2} \boldsymbol{\tau}\right| \psi_{0 i}\right\rangle \\
& \quad+i\left\langle\psi_{0 i}\left|D_{\nu i}\right| \psi_{0 i}\right\rangle\left\langle\psi_{0 j}\left|i g \gamma_{\lambda} \boldsymbol{A}_{\lambda j} \cdot \frac{1}{2} \boldsymbol{\tau}\right| \psi_{0 i}\right\rangle+\mathrm{O}\left(m \frac{1}{d^{2}}, \frac{1}{d^{5}}\right) .
\end{aligned}
$$

Inserting the results (90) and (91) into eq. (87) and using

$$
\begin{aligned}
& \frac{\partial}{\partial z_{\nu i}}\left\langle\psi_{0 i}\left|i g \gamma_{\lambda} \boldsymbol{A}_{\lambda i} \cdot \frac{1}{2} \boldsymbol{\tau}\right| \psi_{0 j}\right\rangle=-\frac{\partial}{\partial z_{\nu i}} B_{j i}, \\
& \frac{\partial}{\partial z_{\nu i}}\left\langle\psi_{0 j}\left|i g \gamma_{\lambda} \boldsymbol{A}_{\lambda i} \cdot \frac{1}{2} \tau\right| \psi_{0 i}\right\rangle=-\frac{\partial}{\partial z_{\nu i}} B_{i j}, \\
& \left\langle\psi_{0 i}\left|D_{\nu i}\right| \psi_{0 i}\right\rangle=-\frac{1}{2}\left\langle\psi_{0 i}\left|\left\{\gamma_{\nu}, D_{i}\right\}\right| \psi_{0 i}\right\rangle=0,
\end{aligned}
$$

we obtain

$$
\begin{aligned}
\frac{\delta \Gamma_{\mathrm{f}}(A)_{\text {ren }}}{\delta z_{i \nu}} & =N_{\mathrm{f}} \sum_{i \neq i}\left[C_{i i} \frac{\partial}{\partial z_{\nu i}} B_{i i}+C_{i i} \frac{\partial}{\partial z_{\nu i}} B_{i j}\right]+\mathrm{O}\left(m, \frac{1}{d^{2}}\right) \\
& =N_{\mathrm{f}} \operatorname{Tr}\left(B^{-1} \frac{\partial}{\partial z_{\nu i}} B\right)+\mathrm{O}\left(m, \frac{1}{d^{2}}\right) .
\end{aligned}
$$

In the limit $1 / d \ll m$, the effective action $\mathrm{I}_{\mathrm{f}}(A)_{\text {ren }}$ should approach the sum of effective actions due to individual (anti-) instantons, viz.

$$
\Gamma_{\mathrm{f}}(A)_{\text {ren }} \underset{1 / d \ll m}{\longrightarrow} \sum_{i=1}^{n}\left[N_{\mathrm{f}} \log m+\Gamma_{\mathrm{f}}^{\prime}\left(A_{\mu i}\right)\right]+\mathrm{O}(m),
$$

where we denoted

$$
\mathrm{e}^{\Gamma_{f}^{\prime}\left(\boldsymbol{A}_{\mu i}\right)}=\left[\frac{\operatorname{det}^{\prime}-i \gamma_{\mu}\left(\partial_{\mu}-i g \boldsymbol{A}_{\mu i} \cdot \frac{1}{2} \tau\right)}{\operatorname{det}-i \gamma_{\mu} \partial_{\mu}}\right]_{\text {ren }} .
$$

(Here, det' indicates that the zero eigenmode is to be deleted from consideration, and for renormalization, see ref. [1].) From eqs. (95) and (96), we can uniquely determine the fermionic effective action in the background gauge field (1);

$$
\Gamma_{\mathrm{f}}(A)_{\text {ren }}=N_{\mathrm{f}} \log (\operatorname{det}-i B)+\sum_{i=1}^{n} \Gamma_{\mathrm{f}}^{\prime}\left(A_{\mu i}\right)+\mathrm{O}\left(m, \frac{1}{d}\right) .
$$

In particular, for an instanton-anti-instanton system, eq. (98) gives

$$
\begin{gathered}
\Gamma_{\mathrm{f}}(A)_{\operatorname{ren}}=N_{\mathrm{f}} \log \left(m^{2}+|a|^{2}\right)+\Gamma_{\mathrm{f}}^{\prime}\left(A_{\mu 1}\right) \\
+\Gamma_{\mathrm{f}}^{\prime}\left(A_{\mu 2}\right)+\mathrm{O}\left(m, \frac{1}{\left|z_{1}-z_{2}\right|}\right),
\end{gathered}
$$


where the constant $a$ is given in eq. (66). We also note that, with the exact classical solution (2) as a single (anti-) instanton potential, 't Hooft evaluated $\Gamma_{\mathrm{f}}\left(A_{\mu i}\right)$ with the result [1]

$$
\Gamma_{\mathrm{f}}^{\prime}\left(A_{\mu i}\right)=N_{\mathrm{f}} \log \rho_{i}-\frac{2}{3} N_{\mathrm{f}} \log \left(\mu_{0} \rho_{i}\right)+2 N_{\mathrm{f}}(0.1458 \ldots) .
$$

Again, the term $N_{\mathrm{f}} \log (\mathrm{det}-i B)$ in eq. (98) has a simple interpretation in terms of the zero-mode approximation $[2,13]$; it is simply the logarithm of the product of all the eigenvalues of the operator (69) in the zero-mode subspace. However, if we had used the zero-mode approximation (68) for the propagation function to calculate the fermion-induced isospin current and the fermionic effective action, we would not have obtained the result (98). (Even the leading term, $N_{\mathrm{f}} \log (\operatorname{det}-i B)$, cannot be obtained this way.) As we have already explained at the end of sect. 2, it is crucial to include interference terms between the zero modes and continuum when both instantons and anti-instantons are present. In other words, the zeromode approximations for the fermionic effective action in such cases are, rather surprisingly, the results obtained after including complicated mixing terms between the zero modes and continuum. We have also shown that there is no undetermined $\mathrm{O}(1)$ term in eq. (98). (This fact is important in the next section, in particular to cstablish cq. (109).) For $m=0$, the term $N_{\mathrm{f}} \log (\operatorname{det}-i B)$ describes logarithmic attractive interactions between instantons and anti-instantons.

\section{Cluster decomposition in massless QCD}

In this section, we study the vacuum structure of QCD with massless fermions, using the fermion propagation function and the fermion-induced effective action determined in previous sections. Without massless fermions in the theory, Callan, Dashen and Gross [2] and also Jackiw and Rebbi [3] showed that the physical vacuum states of $\mathrm{QCD}$ are the so-called $|\theta\rangle$ vacua $(|\theta|<\pi)$. Different values of $\theta$ give inequivalent vacuum states due to quantum mechanical tunnelling between topologically distinct winding number vacua (so called $|n\rangle$ vacua). Of course, such tunelling phenomena may be described by (anti-)instantons in Euclidean path integral language. On the other hand, there is no tunnelling between different winding number vacua with massless fermions present. It is then natural to suspect that the $|\theta\rangle$ vacua in massless $\mathrm{QCD}$ are merely gauge-equivalent copies of the ordinary perturbation theory vacuum $\mid n=0$ ), with no physical significance at all. In the weak coupling approximation and under some plausible assumptions, we shall below show that this is not so. We will first show that the ordinary perturbation theory vacuum in massless QCD cannot be allowed since the cluster decomposition for certain correlation functions is violated. In the $|\theta\rangle$ vacua, the cluster decomposition is restored, and different values of $\theta$ correspond to different vacuum states since certain operators of non-zero chirality obtain different vacuum expectation values. 
Let us imagine that the vacuum in massless QCD corresponds to the ordinary perturbation theory vacuum (i.e., unique vacuum), $|n=0\rangle$. If $\tilde{O}_{ \pm}(x)$ is a local operator of chirality $\pm 2 N_{\mathrm{f}}$, then, with the unique vacuum, we must have

$$
\left\langle n=0\left|\check{O}_{ \pm}(x)\right| n=0\right\rangle=0 .
$$

We now recall that, for any proper vacuum, general quantum field theory requires the cluster decomposition

$$
\left\langle n=0\left|\tilde{O}_{+}\left(x_{1}\right) \tilde{O}_{-}\left(x_{2}\right)\right| n=0\right\rangle \underset{\left|x_{1}-x_{2}\right| \rightarrow \infty}{\longrightarrow}\left\langle n=0\left|\tilde{O}_{+}\left(x_{1}\right)\right| n=0\right\rangle\left\langle n=0\left|\tilde{O}_{-}\left(x_{2}\right)\right| n=0\right\rangle,
$$

and thus, according to eq. (101), the right-hand side should vanish. We will now demonstrate that this correlation function actually approaches a non-vanishing constant asymptotically. For definiteness, we will consider the operators

$$
\tilde{O}_{ \pm}(x)=:\left[\sum_{i=1}^{N_{\mathrm{f}}} \bar{\psi}_{i}(x) \stackrel{1 \pm \gamma_{5}}{2} \psi_{i}(x)\right]^{N_{\mathrm{f}}}:
$$

where the notation: : implies an appropriate subtraction of short-distance infinities in such a way that eq. (101) may be satisfied. Using the Euclidean path integral representation, we have

$$
\begin{aligned}
\langle n & \left.=0\left|\tilde{O}_{+}\left(x_{1}\right) \tilde{O}_{-}\left(x_{2}\right)\right| n=0\right\rangle \\
& =\mathcal{N} \int\left[\mathscr{D} A_{\mu}^{a}\right]_{w=0}[\mathscr{D} \bar{\psi}][\mathscr{D} \psi] \exp \left\{\int \mathrm{d}^{4} x\left[-\frac{1}{4} F_{\mu \nu}^{a} F_{\mu \nu}^{a}+\sum_{i=1}^{N_{f}} i \bar{\psi}_{i} \gamma_{\mu} D_{\mu} \psi_{i}\right]\right\} \\
& \times:\left[\sum_{i=1}^{N_{f}} \psi_{i}\left(x_{1}\right) \frac{1+\gamma_{5}}{2} \psi_{i}\left(x_{1}\right)\right]^{N_{\mathrm{f}}}::\left[\sum_{i=1}^{N_{f}} \psi_{i}\left(x_{2}\right) \frac{1-\gamma_{5}}{2} \psi_{i}\left(x_{2}\right)\right]^{N_{\mathrm{f}}}:,
\end{aligned}
$$

where, for a very weak coupling, we may take the normalization

$$
\begin{aligned}
\mathcal{N}^{-1} & =\int\left[\mathscr{D} A_{\mu}^{a}\right]_{w=0}[\mathscr{D} \bar{\psi}][\mathscr{D} \psi] \\
& \times \exp \left\{\int \mathrm{d}^{4} x\left[-\frac{1}{4}\left(\partial_{\mu} A_{\nu}^{a}-\partial_{\nu} A_{\mu}^{a}\right)\left(\partial_{\mu} A_{\nu}^{a}-\partial_{\nu} A_{\mu}^{a}\right)+\sum_{i=1}^{N_{t}} i \tilde{\psi}_{i} \gamma_{\mu} \partial_{\mu} \psi_{i}\right] .\right.
\end{aligned}
$$

By the assumption about the vacuum, we only include gauge field histories with the total Pontryagin number zero in the path integral (104).

Naively, one may expect that, for $\left|x_{1}-x_{2}\right| \rightarrow \infty$, the correlation function (104) will be $\mathrm{O}\left(1 / x_{1}-\left.x_{2}\right|^{6 N_{f}}\right)$, as the free field theory indicates. This is totally false with the background gauge field of the type

$$
\begin{aligned}
& A_{\mu}^{a}(x)=A_{\mu+}^{a}\left(x-z_{1}\right)+A_{\mu-}^{a}\left(x-z_{2}\right), \\
& z_{1} \approx x_{2}, \quad z_{2} \approx x_{1},
\end{aligned}
$$

where $A_{\mu}^{a}\left(A_{\mu_{-}}^{a}\right)$ describe a localized instanton (anti-instanton) with the center 
$x=z_{1}\left(x=z_{2}\right)$. To see that explicitly, let us consider the simplest case, $N_{\mathrm{f}}=1$, and assume that an arbitrarily large-size instanton (or anti-instanton) does not give a significant contribution to the path integral (104). Then, for sufficiently large $\left|x_{1}-x_{2}\right|$, we may write the total contributions to the path integral (104) due to gauge fields of the type (106) such as

$$
\begin{aligned}
\langle n & \left.=0\left|\tilde{O}_{+}\left(x_{1}\right) \tilde{O}_{-}\left(x_{2}\right)\right| n=0\right\rangle_{+-} \\
& =\left\{\mathcal{N}_{G} \int\left[\mathscr{D} A_{\mu+}^{a}\right]_{w=1} \exp \left[-\frac{1}{4} \int \mathrm{d}^{4} x F_{\mu \nu+}^{a} F_{\mu \nu+}^{a}\right]\right\} \\
& \times\left\{\mathcal{N}_{G} \int\left[\mathscr{D} A_{\mu-}^{a}\right]_{w=-1} \exp \left[-\frac{1}{4} \int \mathrm{d}^{4} x F_{\mu \nu-}^{a} F_{\mu \nu-}^{a}\right]\right\} \\
& \times \mathrm{e}^{\Gamma_{f}\left(A_{\mu}=A_{\mu+}+A_{\mu-}\right)} \operatorname{Tr}\left(\frac{1+\gamma_{5}}{2} S\left(x_{1}, x_{2}\right) \frac{1-\gamma_{5}}{2} S\left(x_{2}, x_{1}\right)\right),
\end{aligned}
$$

where the normalization $\mathscr{N}_{\mathrm{G}}$ is given by

$$
\mathcal{N}_{\mathrm{G}}^{-1}=\int\left[\mathscr{D} A_{\mu}^{a}\right]_{w=0} \exp \left[-\frac{1}{4} \int \mathrm{d}^{4} x\left(\partial_{\mu} A_{\nu}^{a}-\partial_{\nu} A_{\mu}^{a}\right)\left(\partial_{\mu} A_{\nu}^{a}-\partial_{\nu} A_{\mu}^{a}\right)\right]
$$

In eq. (107), $\Gamma_{\mathrm{f}}$ and $S\left(x_{1}, x_{2}\right)$ denote the fermion-induced effective action and fermion propagation function in the instanton-anti-instanton background gauge field (106). Using the explicit expressions (67) and (99) (with $m=0$ ) for $S\left(x_{1}, x_{2}\right)$ and $\Gamma_{\mathrm{f}}$, it is easy to see that the expression (107) approaches a constant asymptotically, i.e.,

$$
\begin{aligned}
& \lim _{\left|x_{1}-x_{2}\right| \rightarrow \infty}\left\langle n=0\left|\tilde{O}_{+}\left(x_{1}\right) \tilde{O}_{-}\left(x_{2}\right)\right| n=0\right\rangle \\
& =\left\{\mathcal{N}_{\mathrm{G}} \int\left[\mathscr{D} A_{\mu+}^{a}\right]_{w=1} \exp \left[-\frac{1}{4} \int \mathrm{d}^{4} x F_{\mu \nu+}^{a} F_{\mu \nu+}^{a}\right] \mathrm{e}^{\Gamma_{\mathrm{r}}^{\prime}\left(A_{\mu+}\right)}\left|\psi_{0+}\left(x_{2}-z_{1}\right)\right|^{2}\right\} \\
& \quad \times\left\{\mathcal{H}_{G} \int\left[\mathscr{D} A_{\mu-}^{a}\right]_{w=-1} \exp \left[-\frac{1}{4} \int \mathrm{d}^{4} x F_{\mu \nu-}^{a} F_{\mu \nu-}^{a}\right] \mathrm{e}^{\Gamma_{\mathrm{f}}^{\prime}\left(A_{\mu-}\right)}\left|\psi_{0-}\left(x_{1}-z_{2}\right)\right|^{2}\right\} \\
& \quad \neq 0
\end{aligned}
$$

where $\psi_{0+}\left(\psi_{0-}\right)$ represents the fermion zero-mode wave function in the field $A_{\mu+}^{a}\left(A_{\mu-}^{a}\right)$.

From eq. (107), we now observe that

$$
\begin{aligned}
& \lim _{\left|x_{1}-x_{2}\right| \rightarrow \infty}\left\langle n-0\left|\tilde{O}_{+}\left(x_{1}\right) \tilde{O}_{-}\left(x_{2}\right)\right| n=0\right\rangle \\
& \quad=\left\langle n=0\left|\tilde{O}_{+}\left(x_{1}\right)\right| n=1\right\rangle\left\langle n=1\left|\tilde{O}_{-}\left(x_{2}\right)\right| n=0\right\rangle
\end{aligned}
$$


where

$$
\begin{aligned}
\langle n & \left.=1\left|\tilde{O}_{-}\left(x_{2}\right)\right| n=0\right\rangle=\mathcal{N} \int\left[\mathscr{D} A_{\mu+}^{a}\right]_{w=1}[\mathscr{D} \bar{\psi}][\mathscr{D} \psi] \\
& \times \exp \left[\int \mathrm{d}^{4} x\left[-\frac{1}{4} F_{\mu \nu+}^{a} F_{\mu \nu+}^{a}+i \bar{\psi} \gamma_{\mu} D_{\mu} \psi\right]\right]: \bar{\psi}\left(x_{2}\right) \frac{1-\gamma_{5}}{2} \psi\left(x_{2}\right):,
\end{aligned}
$$

and $\left\langle n=0\left|\tilde{O}_{+}\left(x_{1}\right)\right| n=1\right\rangle$ is given by an analogous path integral in the $w=-1$ sector. In fact, a straightforward calculation shows that the result $(110)$ is true for all $N_{\mathrm{f}}$. (Note that, to calculate the expectation values of fermion operators in the $w= \pm 1$ sector, the regulated fermion propagator (11), with the approximations (12) and (13), can be used effectively.) We note that a non-vanishing asymptotic value for $\left\langle n=0\left|\tilde{O}_{+}\left(x_{1}\right) \tilde{O}_{-}\left(x_{2}\right)\right| n=0\right\rangle$ has been achieved by a precise balance between the rapidly vanishing fermion determinant $\left[e^{\Gamma_{f}} \propto|a|^{2 N_{\mathrm{f}}}=\mathrm{O}\left(1 /\left|z_{1}-z_{2}\right|^{6 N_{\mathrm{f}}}\right)\right]$ and the rapidly increasing product of fermion propagation functions in the background gauge field (106) $\left[S\left(x_{1}, x_{2}\right) \propto a^{-1}=\mathrm{O}\left(\left|z_{1}-z_{2}\right|^{3}\right)\right]$. Analogous behavior in the Schwinger model has been noted earlier by Nielsen and Schroer [7]. For a weak coupling and if we can ignore arbitrarily large-size (anti-) instantons as we have assumed, one can easily convince oneself that contributions to the path integral (104) due to gauge fields of $n_{+}=n-n_{+} \neq 1$ are negligible in the limit $\left|x_{1}-x_{2}\right| \rightarrow \infty$. Thus, we may conclude that

$$
\begin{aligned}
& \lim _{\left|x_{1}-x_{2}\right| \rightarrow \infty}\left\langle n=0\left|\tilde{O}_{+}\left(x_{1}\right) \tilde{O}_{-}\left(x_{2}\right)\right| n=0\right\rangle \\
& \quad=\left\langle n=0\left|\tilde{O}_{+}\left(x_{1}\right)\right| n=1\right\rangle\left\langle n=1\left|\tilde{O}_{-}\left(x_{2}\right)\right| n=0\right\rangle \\
& \quad \neq 0 .
\end{aligned}
$$

This shows that the cluster decomposition is violated in the ordinary perturbation theory vacuum.

It is well-known that general local quantum field theory relates the failure of the cluster decomposition to degenerate vacua, here, the $\theta$ vacua. We will now show how the cluster decomposition is restored in the $|\theta\rangle$ vacua for the case of $N_{\mathrm{f}}=1$, leaving the cases with more fermion flavours to readers. In the $\theta$ vacua (and with $N_{\mathrm{f}}=1$ ), we have the path integral representations [2]

$$
\begin{aligned}
& \left\langle\theta\left|\tilde{O}_{ \pm}(x)\right| \theta\right\rangle=\mathcal{N} \sum_{n=-\infty}^{\infty} \int\left[\mathscr{D} A_{\mu}^{a}\right]_{w=n}[\mathscr{D} \bar{\psi}][\mathscr{D} \psi] \mathrm{e}^{i n \theta} \\
& \quad \times \exp \left\{\int \mathrm{d}^{4} x\left[-\frac{1}{4} F_{\mu \nu}^{a} F_{\mu \nu}^{a}+i \bar{\psi} \gamma_{\mu} D_{\mu} \psi\right]\right\}: \bar{\psi}(x) \frac{1 \pm \gamma_{5}}{2} \psi(x):,
\end{aligned}
$$


and

$$
\begin{aligned}
& \left\langle\theta\left|\tilde{O}_{+}\left(x_{1}\right) \tilde{O}_{-}\left(x_{2}\right)\right| \theta\right\rangle=\mathscr{N} \sum_{n=-\infty}^{\infty} \int\left[\mathscr{D} A_{\mu}^{a}\right]_{w=n}[\mathscr{D} \bar{\psi}][\mathscr{D} \psi] \mathrm{e}^{i n \theta} \\
& \quad \times \operatorname{cxp}\left\{\int \mathrm{d}^{4} x\left[-\frac{1}{4} F_{\mu \nu}^{a} F_{\mu \nu}^{a}+i \bar{\psi} \gamma_{\mu} D_{\mu} \psi\right]\right\} \\
& \quad \times\left[: \bar{\psi}\left(x_{1}\right) \frac{1+\gamma_{5}}{2} \psi\left(x_{1}\right):: \bar{\psi}\left(x_{2}\right) \frac{1-\gamma_{5}}{2} \psi\left(x_{2}\right):\right]
\end{aligned}
$$

where $\left[\mathscr{D} A_{\mu}^{a}\right]_{w=n}$ implies the integration over gauge field histories with $w=n$. In eq. (113), non-vanishing contributions come from the $w=\mp 1$ sector only, and we thus obtain

$$
\begin{aligned}
& \left\langle\theta\left|\tilde{O}_{ \pm}(x)\right| \theta\right\rangle=\mathrm{e}^{\mp i \theta}\left\{\mathcal{N}_{\mathrm{G}} \int\left[\mathscr{D} A_{\mu \mp}^{a}\right]_{w=\mp 1}\right. \\
& \left.\quad \times \exp \left\{\int \mathrm{d}^{4} x\left[-\frac{1}{4} F_{\mu \nu \mp}^{a} F_{\mu \nu \mp}^{a}+\Gamma_{\mathrm{f}}^{\prime}\left(A_{\mu \mp}\right)\right]\right\}\left|\psi_{0 \mp}(x)\right|^{2}\right\} \\
& \quad \neq 0
\end{aligned}
$$

i.e., the operator $\tilde{O}_{ \pm}$obtains a non-vanishing vacuum expectation value. Similarly, in the limit $\left|x_{1}-x_{2}\right| \rightarrow \infty$, the path integral in eq. (114) obtains a non-vanishing contribution from the $w=0$ sector, and thus

$$
\begin{aligned}
\lim _{\left|x_{1}-x_{2}\right| \rightarrow \infty}\left\langle\theta\left|\tilde{O}_{+}\left(x_{1}\right) \tilde{O}_{-}\left(x_{2}\right)\right| \theta\right\rangle & =\left\langle n=0\left|\tilde{O}_{+}\left(x_{1}\right) \tilde{O}_{-}\left(x_{2}\right)\right| n=0\right\rangle \\
& =\left\langle n=0\left|\tilde{O}_{+}\left(x_{1}\right)\right| n=1\right\rangle\left\langle n=1\left|\tilde{O}_{-}\left(x_{2}\right)\right| n=0\right\rangle
\end{aligned}
$$

where we have used eq. (112). Writing the result (115) such as

$$
\begin{gathered}
\left\langle\theta\left|\tilde{O}_{+}(x)\right| \theta\right\rangle=\mathrm{e}^{-i \theta}\left\langle n=0\left|\tilde{O}_{+}(x)\right| n=1\right\rangle, \\
\left\langle\theta\left|\tilde{O}_{-}(x)\right| \theta\right\rangle=\mathrm{e}^{i \theta}\left\langle n=1\left|\tilde{O}_{-}(x)\right| n=0\right\rangle,
\end{gathered}
$$

it is now clear that eq. (116) implies

$$
\lim _{\left|x_{1}-x_{2}\right| \rightarrow \infty}\left\langle\theta\left|\tilde{O}_{+}\left(x_{1}\right) \tilde{O}_{-}\left(x_{2}\right)\right| \theta\right\rangle=\left\langle\theta\left|\tilde{O}_{+}\left(x_{1}\right)\right| \theta\right\rangle\left\langle\theta\left|\tilde{O}_{-}\left(x_{2}\right)\right| \theta\right\rangle,
$$

i.e., cluster decomposition is satisfied. Different values of $\theta$ correspond to different vacuum states since the operators $\tilde{O}_{ \pm}$have different vacuum expectation values (see eq. (115)). The chiral $\mathrm{U}(1)$ symmetry is explicitly broken by non-vanishing vacuum expectation values for $\tilde{O}_{ \pm}$. However, it has been noted that, despite the fact that the chiral U(1) symmetry is spontaneously broken, we do not have a physical Goldstone boson since a conserved and gauge-invariant chiral charge operator does not exist in the $|\theta\rangle$ vacua due to the axial vector anomaly $[1,2]$. 
Finally we comment on the relation (110). Though it looks like precisely what one would obtain in the dilute-gas type of approximation, the source of the term is quite different. To illustrate this, let us again take $N_{\mathrm{f}}=1$ and introduce the following source terms in the Lagrange function

$$
i J_{+}(x) \bar{\psi}(x) \frac{1+\gamma_{5}}{2} \psi(x)+i J_{-}(x) \bar{\psi}(x) \frac{1-\gamma_{5}}{2} \psi(x) .
$$

Now, the correlation function (104) can be expressed as

$$
\begin{aligned}
\langle n & \left.=0\left|\tilde{O}_{+}\left(x_{1}\right) \tilde{O}_{-}\left(x_{2}\right)\right| n=0\right\rangle \\
& =\mathcal{N}_{\mathrm{G}} \int\left[\mathscr{D A} A_{\mu}^{a}\right]_{w=0} \exp \left[-\frac{1}{4} \int \mathrm{d}^{4} x F_{\mu \nu}^{a} F_{\mu \nu}^{a}\right] \\
& \times\left[\left(-i \frac{\delta}{\delta J_{+}\left(x_{1}\right)}\right)\left(-i \frac{\delta}{\delta J_{-}\left(x_{2}\right)}\right) \frac{\operatorname{det}-i\left(\not D+{ }_{2}^{1}\left(1+\gamma_{5}\right) J_{+}+{ }_{2}^{1}\left(1-\gamma_{5}\right) J_{-}\right)}{\operatorname{det}-i \not \partial}\right]_{J_{+}=J_{-}=0} .
\end{aligned}
$$

For the background gauge field of the type (106) (i.e., an instanton around $x_{2}$ and an anti-instanton around $\left.x_{1}\right)$ and for large $\left|x_{1}-x_{2}\right|$, one may naively approximate the fermion determinant in eq. (121) such as

$$
\begin{aligned}
& \frac{\operatorname{det}-i\left(\not-i g A_{+}-i g A_{-}+\frac{1}{2}\left(1+\gamma_{5}\right) J_{+}+\frac{1}{2}\left(1-\gamma_{5}\right) J_{-}\right)}{\operatorname{det}-i \not \partial} \\
& \simeq \frac{\operatorname{det}-i\left(\not \partial-i g A_{+}+\frac{1}{2}\left(1-\gamma_{5}\right) J_{-}\right)}{\operatorname{det}-i \not \partial} \frac{\operatorname{det}-i\left(\not \partial-i g A_{-}+\frac{1}{2}\left(1+\gamma_{5}\right) J_{+}\right)}{\operatorname{det}-i \not \partial} \\
& =-\left\langle\psi_{0+}\left|J_{-}\right| \psi_{0+}\right\rangle\left\langle\psi_{0-}\left|J_{+}\right| \psi_{0-}\right\rangle \mathrm{e}^{\Gamma_{\mathrm{f}}^{(}\left(A_{\mu+}\right)} \mathrm{e}^{\Gamma_{\mathrm{f}}^{\prime}\left(A_{\mu-}\right)}\left[1+\mathrm{O}\left(J_{+}, J_{-}\right)\right] .
\end{aligned}
$$

(This is the dilute-gas approximation.) Upon differentiating the expression (122) with respect to sources $J_{+}$and $J_{-}$, one immediately obtains the result (109). However, this is not correct. Note that, in eq. (122), $\left\langle\psi_{0_{+}}\left|J_{-}\right| \psi_{0_{+}}\right\rangle$(or $\left.\left\langle\psi_{0_{-}-}\left|J_{+}\right| \psi_{0_{-}}\right\rangle\right)$ takes the place of the zero eigenvalue for the fermion determinant in the instanton (or anti-instanton) field. But, external sources should be considered as infinitesimal (compared to any other parameters in the problem), and the approximation (122) will thus make sense only if $D$ possesses two exact zero eigenmodes. This is not the case in the instanton-anti-instanton system: here, the fermion zero modes in the instanton field gain "small energy" (and join the continuum) by influence of the anti-instanton (rather than $J_{-}$), and vice versa. This makes the interaction of massless fermions with instantons strongly non-local, and one must proceed by calculating the fermion propagation function and effective action as we have done in the present paper. In fact, the whole question of the cluster decomposition in the $|\theta\rangle$ vacua depends on whether these strongly non-local interactions in the $w=0$ sector give the same contribution to $\left\langle 0\left|\tilde{O}_{+}\left(x_{1}\right) \tilde{O}_{-}\left(x_{2}\right)\right| 0\right\rangle$ as the product of local contributions in the $w=1$ and $w=-1$ sectors. This, we have shown in this section. 
We would like to acknowledge useful discussions with Professors L.S. Brown, R.D. Carlitz, and M.B. Einhorn. One of us (C.L.) wishes to thank the Theoretical Physics Department of Fermi National Accelerator Laboratory for hospitality during the summer of 1978 .

\section{Appendix}

Let us consider the gauge potential of the form

$$
A_{\mu}^{a}(x)=\frac{1}{g} \eta_{\mu \nu a}^{(-)} \frac{2 x_{\nu}}{R^{2}} H(R),
$$

where $R=\left(x^{2}\right)^{1 / 2}$. For the gauge potential (A.1), a straightforward calculation yields the classical gauge field action

$$
S_{\mathrm{cl}}=-\frac{48 \pi^{2}}{g^{2}} \int_{-\infty}^{\infty} \mathrm{d} t\left[\frac{1}{2}\left(\frac{\mathrm{d} H}{\mathrm{~d} t}\right)^{2}+\left(H-H^{2}\right)^{2}\right],
$$

and the Pontryagin number

$$
\begin{aligned}
w & =-6 \int_{-\infty}^{\infty} \mathrm{d} t \frac{\mathrm{d} H}{\mathrm{~d} t}\left(H-H^{2}\right) \\
& =-\left.6\left(\frac{1}{2} H^{2}-\frac{1}{3} H^{3}\right)\right|_{R=0} ^{R=\infty},
\end{aligned}
$$

where we used the variable $t=\log R$. To have a finite action, it is evident from eq. (A.2) that the values of $H(R=\infty)$ and $H(R=0)$ should be either 0 or 1 . Typical shapes of the function $H$ corresponding to some interesting field configurations are shown in fig. 1; fig. 1a corresponds to an instanton in the singular gauge, fig. $1 \mathrm{~h}$ to an anti-instanton in the regular gauge, and fig. 1c to the superposition of the two.

We are interested in normalizable solutions to the Dirac equation

$$
\gamma_{\mu}\left(\partial_{\mu}-i \eta_{\mu \nu a}^{(-)} \tau_{a} \frac{x_{\nu}}{R^{2}} H(R)\right) \psi=0 .
$$

It is a simple matter to show that, for any radial function $H(R)$ with $H(R=0)=1$ and $H(R=\infty)=0$ (fig. 1a), eq. (A.4) possesses a normalizable solution which is proportional to

$$
\psi_{0+}(x)=\left[\exp 3 \int^{R} \frac{H\left(R^{\prime}\right)-1}{R^{\prime}} \mathrm{d} R^{\prime}\right] \frac{\tau_{\mu}^{(+)} x_{\mu}}{R} \chi_{+},
$$

where the constant state vector $\chi_{+}$is specified by eq. (6) in the main text. Note that, for large $R$, the zero-mode wave function (A.5) behaves like $\mathrm{O}\left(1 / \boldsymbol{R}^{3}\right.$ ) regardless of a detailed shape of the function $H(R)$. We have assumed this asymptotic behavior in eq. (9) of the main text. Similarly, in a gauge potential with 

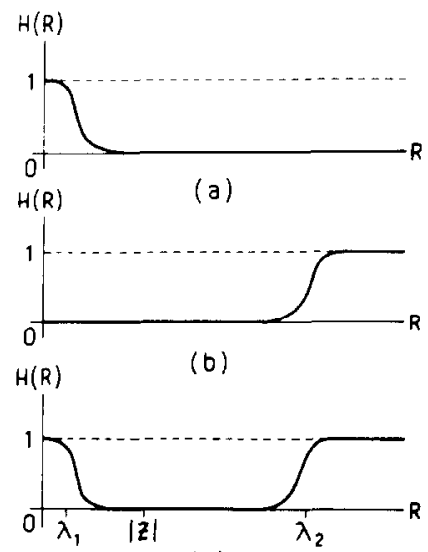

(c)

Fig. 1. Shapes of the function $H$ for (a) an instanton, (b) an anti-instanton and (c) an instanton-anti-instanton system.

$H(R=0)=0$ and $H(R=\infty)=1$ (fig. 1b), there exists a normalizable solution which is proportional to

$$
\psi_{0-}(x)=\left[\exp -3 \int^{R} \frac{H\left(R^{\prime}\right)}{R^{\prime}} \mathrm{d} R^{\prime}\right] \chi_{-},
$$

with the constant vector $\chi$ - given by eq. (6).

To study the Dirac equation (A.4) with an instanton-anti-instanton system shown in fig. $1 \mathrm{c}$, we first note that the partial wave ( $f$ and $g$ denote arbitrary radial functions)

$$
\psi=g(R) R^{-3 / 2} \frac{\tau_{\mu}^{(+)} x_{\mu}}{R} \chi_{+}+f(R) R^{-3 / 2} \chi_{-}
$$

is form invariant under the operation of $\gamma_{\mu} D_{\mu}$ with the gauge potential (A.1). Explicitly,

$$
\begin{aligned}
& \gamma_{\mu} D_{\mu} \psi=\left[\frac{\mathrm{d} f}{\mathrm{~d} R}+\frac{3\left(H-\frac{1}{2}\right)}{R} f\right] R^{-3 / 2} \frac{\tau_{\mu}^{(+)} x_{\mu}}{R} \chi_{+} \\
& +\left[-\frac{\mathrm{d} g}{\mathrm{~d} R}+\frac{3\left(H-\frac{1}{2}\right)}{R} g\right] R^{-3 / 2} \chi-.
\end{aligned}
$$

Since both zero modes given in eqs. (A.5) and (A.6) belong to the partial wave (A.7), we may now conclude that, if there exists an exact zero mode in a widelyseparated instanton-anti-instanton system shown in fig. 1c, it must occur in the 
partial wave (A.7) with the functions $f, g$ satisfying

$$
\frac{\mathrm{d} f}{\mathrm{~d} R}+\frac{3\left(H-\frac{1}{2}\right)}{R} f=0,
$$

and

$$
-\frac{\mathrm{d} g}{\mathrm{~d} R}+\frac{3\left(H-\frac{1}{2}\right)}{R} f=0 .
$$

With $H(R=0)$ and $H(R=\infty)=1$ (see fig. 1c), it is a simple matter to show that there is no bounded solution to eqs. (A.9) and (A.10). This illustrates the absence of normalizable fermion zero mode in the spherical-shell-like configuration shown in fig. 1c; a singular instanton at the origin and a regular anti-instanton at $R=\infty$. By performing a conformal inversion around $x_{\mu}=z_{\mu}\left(\lambda_{2} \gg|z| \gg \lambda_{1}\right.$, see fig. 1c) with the inversion radius $\rho\left(\lambda_{2} \gg \rho \gg|z| \gg \lambda_{1}\right)$, this spherical-shell-like configuration can be changed into the widely separated instanton-anti-instanton system in the singular gauge [12]. Since the Dirac cquation (A.4) is invariant under the conformal inversion, the above example also shows the absence of normalizable fermion zero mode in that conformally inverted system.

\section{References}

[1] G. 't Hooft, Phys. Rev. D14 (1976) 3432; Phys. Rev. Lett. 37 (1976) 8

[2] C.G. Callan, R. Dashen and D.J. Gross, Phys. Lett. 63B (1976) 334.

[3] R. Jackiw and C. Rebbi, Phys. Rev. Lett. 37 (1976) 72.

[4] L.S. Brown, R.D. Carlitz and C. Lee, Phys. Rev. D16 (1977) 417; J. Kiskis, Phys. Rev. D15 (1977) 2329.

[5] C.G. Callan, R. Dashen and D.J. Gross, Phys. Rev. D17 (1978) 2717.

[6] J. Schwinger, Phys. Rev. 128 (1962) 2425; J.H. Lowenstein and J.A. Swieca, Ann. of Phys. 68 (1971) 172.

[7] N.K. Nielsen and B. Schroer, Phys. Lett. 66B (1977) 373; Nucl. Phys. B120 (1977) 62.

[8] A. Belavin, A. Polyakov, A. Schwartz and Y. Tyupkin, Phys. Lett. 59B (1975) 85.

[9] L.S. Brown, R.D. Carlitz, D.B. Creamer and C. Lee, Phys. Rev. D17 (1978) 1583.

[10] L.S. Brown and C. Lee, Phys. Rev. D18 (1978) 2180.

[11] L.S. Brown and D.B. Creamer, Phys. Rev. D18 (1978) 3695.

[12] R. Jackiw and C. Rebbi, Phys. Rev. D14 (1976) 517.

[13] W.F. Palmer and S.S. Pinsky, Ohio State University preprint (1978). 